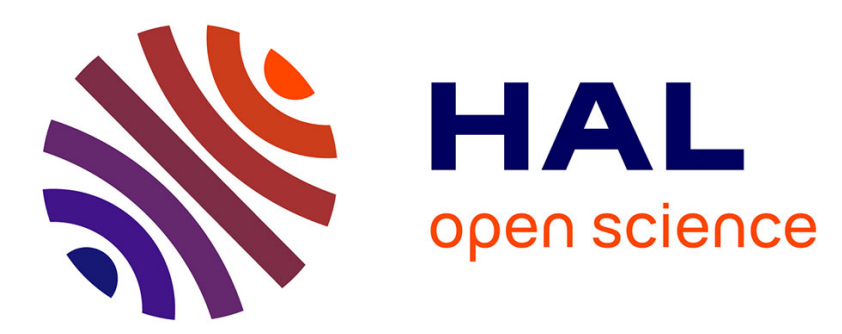

\title{
Quadratic prismatic and hexahedral solid-shell elements for geometric nonlinear analysis of laminated composite structures
}

\author{
Peng Wang, Hocine Chalal, Farid Abed-Meraim
}

\section{- To cite this version:}

Peng Wang, Hocine Chalal, Farid Abed-Meraim. Quadratic prismatic and hexahedral solid-shell elements for geometric nonlinear analysis of laminated composite structures. Composite Structures, 2017, 172, pp.282-296. 10.1016/j.compstruct.2017.03.091 . hal-02386368

\section{HAL Id: hal-02386368 \\ https://hal.science/hal-02386368}

Submitted on 29 Nov 2019

HAL is a multi-disciplinary open access archive for the deposit and dissemination of scientific research documents, whether they are published or not. The documents may come from teaching and research institutions in France or abroad, or from public or private research centers.
L'archive ouverte pluridisciplinaire HAL, est destinée au dépôt et à la diffusion de documents scientifiques de niveau recherche, publiés ou non, émanant des établissements d'enseignement et de recherche français ou étrangers, des laboratoires publics ou privés. 


\title{
Quadratic prismatic and hexahedral solid-shell elements for geometric nonlinear analysis of laminated composite structures
}

\author{
Peng Wang, Hocine Chalal, Farid Abed-Meraim* \\ LEM3, UMR CNRS 7239 - Arts et Métiers ParisTech, 4, rue Augustin Fresnel, 57078 Metz Cedex \\ 03, France
}

\begin{abstract}
The current contribution proposes two quadratic, prismatic and hexahedral, solid-shell elements for the geometric nonlinear analysis of laminated composite structures. The formulation of the proposed solid-shell elements is based on a fully three-dimensional approach combining the assumed-strain method and the reduced-integration technique. In particular, only translational degrees of freedom are considered in the formulation and a preferential direction is chosen as the thickness direction, along which an arbitrary number of integration points are arranged. Making use of different physical local frames, these elements are coupled with fully three-dimensional orthotropic constitutive equations, which allows modeling multilayered composite structures with only a single element layer through the thickness. A series of popular nonlinear benchmark tests for laminated composite structures is performed to assess the performance of the proposed SHB elements. Compared to reference solutions taken from the literature, the results provided by the SHB elements show excellent agreement. Moreover, on the whole, the proposed SHB elements perform better than state-of-the-art ABAQUS elements, which have the same geometry and kinematics, using comparable mesh discretizations.
\end{abstract}

Keywords: quadratic solid-shell elements, assumed-strain method, geometric nonlinearities, orthotropic elasticity, composite structures.

* Corresponding author. Tel.: +(33) 3.87.37.54.79; fax: +(33) 3.87.37.54.70.

E-mail address: farid.abed-meraim@ensam.eu (F. Abed-Meraim). 


\section{Introduction}

In recent years, composite materials have been increasingly employed in modern industries due to their excellent characteristics, which result from the smart combination of superior strength, high stiffness and lightweight. Considering these attractive properties, designers tend to use composite materials as alternative to conventional sheet metals for thin structures to reduce the product weight, while maintaining high mechanical performance. Among various types of composite materials, the fiber-reinforced composite laminates particularly attracted researchers' attention. Thanks to the continuous development of composite manufacturing techniques, laminated composite materials have become among the most favorite advanced materials. Such laminated structures can be used to satisfy various engineering requirements, through the combination of different stacking sequences. In this field, much effort has been devoted to the numerical analysis of laminated composite materials and structures. In particular, extensive research work has been conducted in the literature to establish theoretical solutions or to develop efficient numerical methods for solving nonlinear composite structural problems.

Early investigations mainly focused on the theoretical analysis of simple laminated plates. For instance, Srinivas and Rao [1] proposed a unified exact solution for bending, vibration and buckling of thick orthotropic rectangular plates and laminates. Constrained by the computational resources of that time, they only considered the small strain framework on the basis of Reissner-Mindlin's thick plate theory. Concurrently, Pagano [2], Sciuva [3] and Khdeir [4] derived the exact solutions for composite laminates from the classic Kirchhoff theory. One can also find in the literature other numerical approaches for the analysis of composite structures, such as the spline interpolation method proposed by Cheung and Kong [5], the meshless method adopted by Wang et al. [6], or the global-local higher-order theory developed by $\mathrm{Wu}$ and Chen [7].

Subsequently, other researchers directed attention towards the development of efficient composite shell finite elements. Based on the Lagrangian formulation, Wagner and Gruttmann [8] proposed a simple shell finite element, which is very attractive both for static and dynamic nonlinear analysis of composite structures. Polit and Touratier [9] developed a higher-order triangular finite element for linear and nonlinear analyses of multilayered plates, in which they applied the Argyris interpolation for the consideration of the transverse displacement and the Ganev interpolation for the membrane displacements. Khare et al. [10] established a higher-order 
facet shell element, based on the higher-order shear deformation theory, for vibration analysis of composite and sandwich laminates. Lee and Han [11] developed a 9-node shell element, combining the assumed natural strain (ANS) method and Mindlin-Reissner's theory, for forced vibration analysis of orthotropic and laminated composite materials. In the work of Arciniega and Reddy [12], a higher-order tensor-based shell element formulation has been proposed using Lagrangian interpolation in order to avoid membrane and shear locking for typical laminated composite problems. In conjunction with the Asymptotic Numerical Method [13], Hu et al. [14, 15] proposed a one-dimensional finite element for the analysis of sandwich beams considering layer-wise higher-order transversal displacements. Nguyen-Van et al. [16] used a strain smoothing technique in the formulation of a quadrilateral flat shell element, which was applied to the analysis of buckling and vibration for composite structures. A seven-parameter highpolynomial order continuum shell element was developed by Payette and Reddy [17] for the simulation of the mechanical response of composite shell structures, which requires displacement degrees of freedom (DOF) only and fully three-dimensional constitutive equations. More recently, Choi [18] proposed a geometrically nonlinear shell finite element based on a doubly curved shell theory and von Karman's large deflection theory for the dynamic/impact analysis of laminates. Yu et al. [19] developed a two-dimensional finite element model to investigate the instability phenomena of sandwich plates using the classical Kirchhoff assumptions in the faces and enriched kinematics in the core. Yang et al. [20] proposed a new family of one-dimensional finite elements for wrinkling analysis of thin films using Euler-Bernoulli's kinematics and Carrera's unified formulation [21]. Huang et al. [22] adopted a Fourier-based finite element model to study the instability phenomena of sandwich plates.

From the above literature review, one can conclude that a number of conventional or improved degenerated shell elements are capable of simulating various types of laminated composite structures, due to their high efficiency and accuracy. However, some idealized assumptions are sometimes made in their formulation, which may limit their use in some particular composite structural applications. Also, some locking phenomena, such as shear locking or membrane locking, are often encountered in thin shell applications, thus compromising the overall accuracy and efficiency of the simulations. In order to circumvent these locking phenomena, special numerical treatments are required within these shell formulations, which makes their numerical implementation even more complex. Furthermore, many shell elements are not able to account 
for thickness variation, and they cannot be directly combined with continuum solid elements, due to the complex shell-type kinematics. Consequently, for some particular complex structures, transition elements should be developed to ensure the connection between bulk and structural zones (see, e.g., Liao et al. [23]).

To remedy the above-mentioned limitations, a new alternative approach for modeling thin laminated structures consists in the development of solid-shell elements, in which only displacement DOF are involved. The solid-shell concept combines both the advantages of the 3D formulation of solid elements and the desirable behavior of traditional shell elements. These advantages make the calculation of thickness variation very easy, as well as the connection with conventional solid elements. Similar to classical solid and shell formulations, solid-shell elements are also likely to suffer from various locking phenomena (e.g., membrane, shear, volumetric, thickness locking...). The latter are generally eliminated by resorting to different numerical strategies, such as the reduced integration technique (RI), the assumed strain method (ASM), the enhanced assumed strain method (EAS) or the assumed natural strain method (ANS). During the last decades, much effort has been devoted to the development of efficient solid-shell elements. Klinkel et al. [24] proposed a three-dimensional shell element for the nonlinear analysis of laminated structures, in which the ASM method was used to eliminate the membrane and shear locking phenomena. A low-order solid-shell element was also developed by Vu-Quoc and Tan [25] for the modeling of multilayer shell structures, which combines the EAS and ANS methods in order to avoid locking effects. Quy and Matzenmiller [26] combined both the higher-order shear deformation theory and the EAS method in their solid-shell formulation, so that to improve the transverse shear behavior. On the other hand, Naceur et al. [27] also adopted the combination of EAS and ANS methods to improve the behavior of the low-order hexahedral solid-shell element, which was then applied to the modeling of composite multilayered structures. More recently, Hajlaoui et al. [28] developed a new solid-shell element, using the EAS method and the first-order shear deformation concept, for the buckling analysis of functionally graded materials, while Kpeky et al. [29] proposed an assumed-strain based solid-shell element for the modeling of viscoelastic sandwich structures.

In this paper, two quadratic prismatic and hexahedral solid-shell elements are proposed for the modeling of multilayered composite structures. These elements belong to a class of solid-shell (SHB) elements that have been originally developed by Abed-Meraim and co-workers [30-34]. 
This SHB element family consists of linear prismatic and hexahedral elements (SHB6 and SHB8PS) and their quadratic counterparts (SHB15 and SHB20). These SHB elements are based on a fully three-dimensional formulation with an in-plane reduced-integration scheme and an arbitrarily number of integration points along the thickness direction, which allows modeling thin structures with only a single element layer. In the earlier works on the quadratic SHB elements (i.e., SHB15 and SHB20), their formulation was restricted to the small strain framework, with applications limited to linear isotropic elastic benchmark problems [33]. In the current contribution, however, the quadratic SHB15 and SHB20 solid-shell formulations are extended to the nonlinear analysis of orthotropic materials, which allows the three-dimensional modeling of multilayered composite structures with only a single element layer through the thickness.

The paper is organized as follows. In Section 2, the general formulation of the proposed quadratic solid-shell elements is presented. Then, the performance of the resulting SHB elements is assessed, in Section 3, through a variety of nonlinear benchmark problems for composite laminates. Finally, the main conclusions and remarks are drawn in Section 4.

\section{Formulation of the quadratic solid-shell (SHB) elements}

Owing to several similarities in the formulation of the prismatic and the hexahedral solid-shell elements (i.e., SHB15 and SHB20, respectively), a unified formulation for both solid-shell elements is presented in this section, for the sake of conciseness. This general formulation was originally developed by Abed-Meraim et al. [33], within the framework of small strains and linear isotropic elasticity, while it is extended in the current work to the large strain framework and composite-type anisotropic behavior.

\subsection{Geometry and integration points}

Fig. 1 illustrates the three-dimensional geometry of the 15 -node prismatic solid-shell element (SHB15) and the 20-node hexahedral solid-shell element (SHB20), as well as the location of their integration points. A special direction, denoted by $\zeta$, is chosen as the thickness direction, along which an arbitrary number of Gauss integration points may be arranged. Such a strategy is generally very convenient for thin structures, and particularly for laminated composite structures, for which the whole thickness is modeled with only a single element layer. 


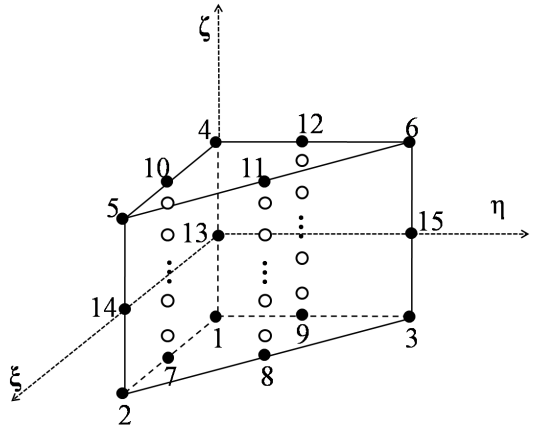

(a) SHB15

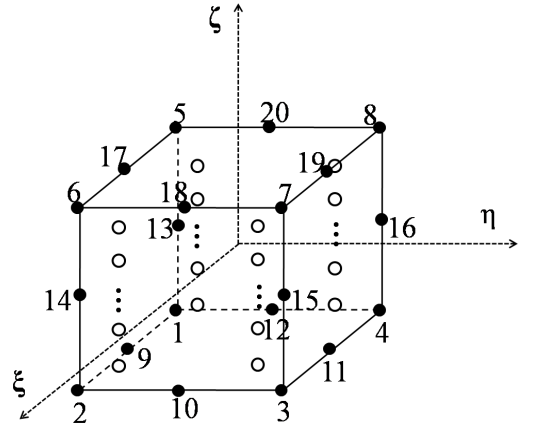

(b) SHB20

Fig. 1. Reference geometry and location of integration points for the SHB15 and SHB20 elements.

\subsection{Kinematic fields and discrete gradient operator}

As mentioned in the introduction, the SHB elements have only displacements as degrees of freedom at their nodes. Accordingly, the spatial coordinates $x_{i}$ and the displacement field $u_{i}$ are interpolated within the element using the classical shape functions for quadratic prismatic and hexahedral elements:

$x_{i}=x_{i I} N_{I}(\xi, \eta, \zeta)=\sum_{I=1}^{n} x_{i I} N_{I}(\xi, \eta, \zeta)$

$u_{i}=d_{i I} N_{I}(\xi, \eta, \zeta)=\sum_{I=1}^{n} d_{i I} N_{I}(\xi, \eta, \zeta)$

where the lowercase subscript $i$ varies from 1 to 3 , and represents the spatial coordinate directions, while the uppercase subscript $I$ goes from 1 to $n$, with $n$ being the number of element nodes ( $n=15$ for the SHB15 element, and $n=20$ for the SHB20 element).

Combining the above equations with the expression of the shape functions leads to an expansion of the displacement field which, when evaluated at the element nodes, can be written in the following form:

$\mathbf{d}_{i}=a_{0 i} \mathbf{s}+a_{1 i} \mathbf{x}_{1}+a_{2 i} \mathbf{x}_{2}+a_{3 i} \mathbf{x}_{3}+\sum_{\alpha} c_{\alpha i} \mathbf{h}_{\alpha}, \quad i=1,2,3$

where $\mathbf{d}_{i}^{T}=\left(d_{i 1}, d_{i 2}, d_{i 3}, \cdots, d_{i n}\right)$ represent the nodal displacement vectors, while $\mathbf{x}_{i}^{T}=\left(x_{i 1}, x_{i 2}, x_{i 3}, \cdots, x_{i n}\right)$ are the nodal coordinate vectors. Note that in Eq. (3) above, there is summation over index $\alpha$, with $\alpha$ ranging from 1 to 11 for the SHB15 element, and from 1 to 16 
for the SHB20 element. Also, vector $\mathbf{s}^{T}=(1,1, \cdots, 1)$ is a fifteen-component constant vector in the case of the SHB15 element, and a twenty-component constant vector for the SHB20 element. As to vectors $\mathbf{h}_{\alpha}$, they are obtained by evaluating some $h_{\alpha}$ functions at the element nodes. These involved $h_{\alpha}$ functions, whose components are functions of the nodal coordinates $(\xi, \eta, \zeta)$ in the reference coordinate system, are defined for the SHB15 element as:

$\left\{\begin{array}{l}h_{1}=\xi \zeta, h_{2}=\eta \zeta, h_{3}=\xi \eta, h_{4}=\xi \eta \zeta, h_{5}=\xi^{2}, h_{6}=\eta^{2}, h_{7}=\zeta^{2} \\ h_{8}=\xi^{2} \zeta, h_{9}=\eta^{2} \zeta, h_{10}=\xi \zeta^{2}, h_{11}=\eta \zeta^{2}\end{array}\right.$

while for the SHB20 element, their expressions are given by:

$$
\left\{\begin{array}{l}
h_{1}=\xi \zeta, h_{2}=\eta \zeta, h_{3}=\xi \eta, h_{4}=\xi^{2}, h_{5}=\eta^{2}, h_{6}=\zeta^{2}, h_{7}=\xi \eta \zeta, \\
h_{8}=\xi^{2} \eta, h_{9}=\xi^{2} \zeta, h_{10}=\eta^{2} \xi, h_{11}=\eta^{2} \zeta, h_{12}=\xi \zeta^{2}, h_{13}=\eta \zeta^{2}, \\
h_{14}=\xi^{2} \eta \zeta, h_{15}=\xi \eta^{2} \zeta, h_{16}=\xi \eta \zeta^{2} .
\end{array}\right.
$$

By applying some preliminarily established orthogonality conditions, and introducing the Hallquist [35] vectors $\mathbf{b}_{i}=\frac{\partial \mathbf{N}}{\partial x_{i \mid \xi=\eta=\zeta=0}}$, with $\mathbf{N}$ the vector whose components are the shape functions $N_{I}$, the expressions of the unknown constants $a_{j i}$ and $c_{\alpha i}$ in Eq. (3) are derived as:

$a_{j i}=\mathbf{b}_{j}^{T} \cdot \mathbf{d}_{i}, \quad c_{\alpha i}=\boldsymbol{\gamma}_{\alpha}^{T} \cdot \mathbf{d}_{i}$,

where the detailed expressions of vectors $\gamma_{\alpha}$ for the SHB15 and SHB20 elements can be found in [33].

Then, the components of the linear part of the strain tensor $\varepsilon$ within the element can be easily expressed as:

$\varepsilon_{i j}=\frac{1}{2}\left(d_{i I} N_{I, j}+d_{j I} N_{I, i}\right)$

where, again, the lowercase subscripts $i$ and $j$ range from 1 to 3 , while the uppercase subscript $I$ goes from 1 to $n$. The linear part of the strain field given in Eq. (7), which is defined as the symmetric part of the displacement gradient $u_{i, j}$, can be rewritten in a vector form as follows: 
$\nabla_{s}(\mathbf{u})=\left[\begin{array}{c}u_{x, x} \\ u_{y, y} \\ u_{z, z} \\ u_{x, y}+u_{y, x} \\ u_{y, z}+u_{z, y} \\ u_{x, z}+u_{z, x}\end{array}\right]=\mathbf{B} \cdot \mathbf{d}=\mathbf{B} \cdot\left[\begin{array}{c}\mathbf{d}_{x} \\ \mathbf{d}_{y} \\ \mathbf{d}_{z}\end{array}\right]$

where the discrete gradient operator $\mathbf{B}$ takes the following matrix form:

$$
\mathbf{B}=\left[\begin{array}{ccc}
\mathbf{b}_{x}^{T}+h_{\alpha, x} \boldsymbol{\gamma}_{\alpha}^{T} & \mathbf{0} & \mathbf{0} \\
\mathbf{0} & \mathbf{b}_{y}^{T}+h_{\alpha, y} \boldsymbol{\gamma}_{\alpha}^{T} & \mathbf{0} \\
\mathbf{0} & \mathbf{0} & \mathbf{b}_{z}^{T}+h_{\alpha, z} \boldsymbol{\gamma}_{\alpha}^{T} \\
\mathbf{b}_{y}^{T}+h_{\alpha, y} \boldsymbol{\gamma}_{\alpha}^{T} & \mathbf{b}_{x}^{T}+h_{\alpha, x} \boldsymbol{\gamma}_{\alpha}^{T} & \mathbf{0} \\
\mathbf{0} & \mathbf{b}_{z}^{T}+h_{\alpha, z} \boldsymbol{\gamma}_{\alpha}^{T} & \mathbf{b}_{y}^{T}+h_{\alpha, y} \boldsymbol{\gamma}_{\alpha}^{T} \\
\mathbf{b}_{z}^{T}+h_{\alpha, z} \boldsymbol{\gamma}_{\alpha}^{T} & \mathbf{0} & \mathbf{b}_{x}^{T}+h_{\alpha, x} \boldsymbol{\gamma}_{\alpha}^{T}
\end{array}\right] .
$$

Note that in Eqs. (7) and (9) above, the convention of implied summation over the repeated indices $I$ and $\alpha$, respectively, has been used.

\subsection{Variational principle}

The $\mathrm{Hu}-$ Washizu mixed variational principle, in conjunction with the updated Lagrangian approach, is used in this work to construct the assumed-strain formulations of the SHB15 and the SHB20 solid-shell elements. More specifically, the current assumed-strain formulations are based on the simplified form of this three-field variational principle, as suggested by Simo and Hughes [36], which writes

$\delta \pi(\dot{\overline{\boldsymbol{\varepsilon}}})=\int_{\Omega_{e}} \delta \dot{\overline{\boldsymbol{\varepsilon}}}^{T} \cdot \boldsymbol{\sigma} d \Omega-\delta \dot{\mathbf{d}}^{T} \cdot \mathbf{f}^{e x t}=0$,

where $\delta$ denotes a variation, $\dot{\overline{\boldsymbol{\varepsilon}}}$ the assumed-strain rate, $\boldsymbol{\sigma}$ the Cauchy stress tensor, $\dot{\mathbf{d}}$ the nodal velocities, and $\mathbf{f}^{\text {ext }}$ the external nodal forces.

The expression of the assumed-strain rate is given by

$\dot{\overline{\boldsymbol{\varepsilon}}}(x, t)=\overline{\mathbf{B}} \cdot \dot{\mathbf{d}}$, 
where matrix $\overline{\mathbf{B}}$ is derived from the classical discrete gradient operator $\mathbf{B}$ through a projection technique, in the aim of eliminating most locking phenomena (e.g., membrane locking, shear locking, thickness locking, etc.).

Substituting Eq. (11) into the simplified form of the $\mathrm{Hu}-$ Washizu variational principle, and taking the nonlinear part of the strain field into consideration, the expressions of the element stiffness matrix and internal force vector are obtained as follows:

$\mathbf{K}_{e}=\int_{\Omega_{e}} \overline{\mathbf{B}}^{T} \cdot \mathbf{C}(\theta) \cdot \overline{\mathbf{B}} d \Omega+\mathbf{K}_{\text {geom }}, \quad \mathbf{f}^{\mathrm{int}}=\int_{\Omega_{e}} \overline{\mathbf{B}}^{T} \cdot \boldsymbol{\sigma}(\dot{\overline{\mathbf{\varepsilon}}}) d \Omega$,

where $\mathbf{C}(\theta)$ is the fourth-order tangent modulus describing the orthotropic elastic behavior of the material, whose expression will be detailed in the subsequent subsection, and $\mathbf{K}_{\text {geom }}$ is the geometric stiffness matrix, which originates from the nonlinear (quadratic) part of the strain tensor.

It is worth noting that, because no noticeable locking has been observed when evaluating the proposed elements on selective benchmark problems, no projection has been applied to their discrete gradient operator. Additionally, the analysis of stiffness matrix rank deficiency did not reveal any potential zero-energy hourglass modes and, accordingly, no stabilization against spurious kinematic modes is required for the proposed quadratic solid-shell elements. These are major differences with the linear versions of the SHB elements, since no additional treatments are required in the formulation of the proposed quadratic SHB15 and SHB20 elements, thereby simplifying their numerical implementation.

\subsection{Definition of the local frames for the SHB solid-shell elements}

In order to extend the earlier formulations of the quadratic SHB elements to the framework of large strains and orthotropic elastic behavior for composite materials, two local frames are defined with respect to the global coordinate system. Each local frame is orthogonal and is identified by its rotation matrix $\mathbf{R}$, which allows transferring the stress / strain state and all internal variables of the element from the local frame to the global one, and vice versa. Fig. 2 illustrates the local frames used in the formulation of the proposed SHB elements. The first local frame, denoted as the "element frame", is attached to the element mid-plane associated with each integration point. In such an element frame, the fourth-order orthotropic elasticity tensor $\mathbf{C}(\theta)$ is 
specified for each ply that constitutes the composite laminate, with $\theta$ being the fiber orientation angle with respect to the orthotropy directions. The second local frame is the so-called "material frame", in which the constitutive equations of the material are formulated.

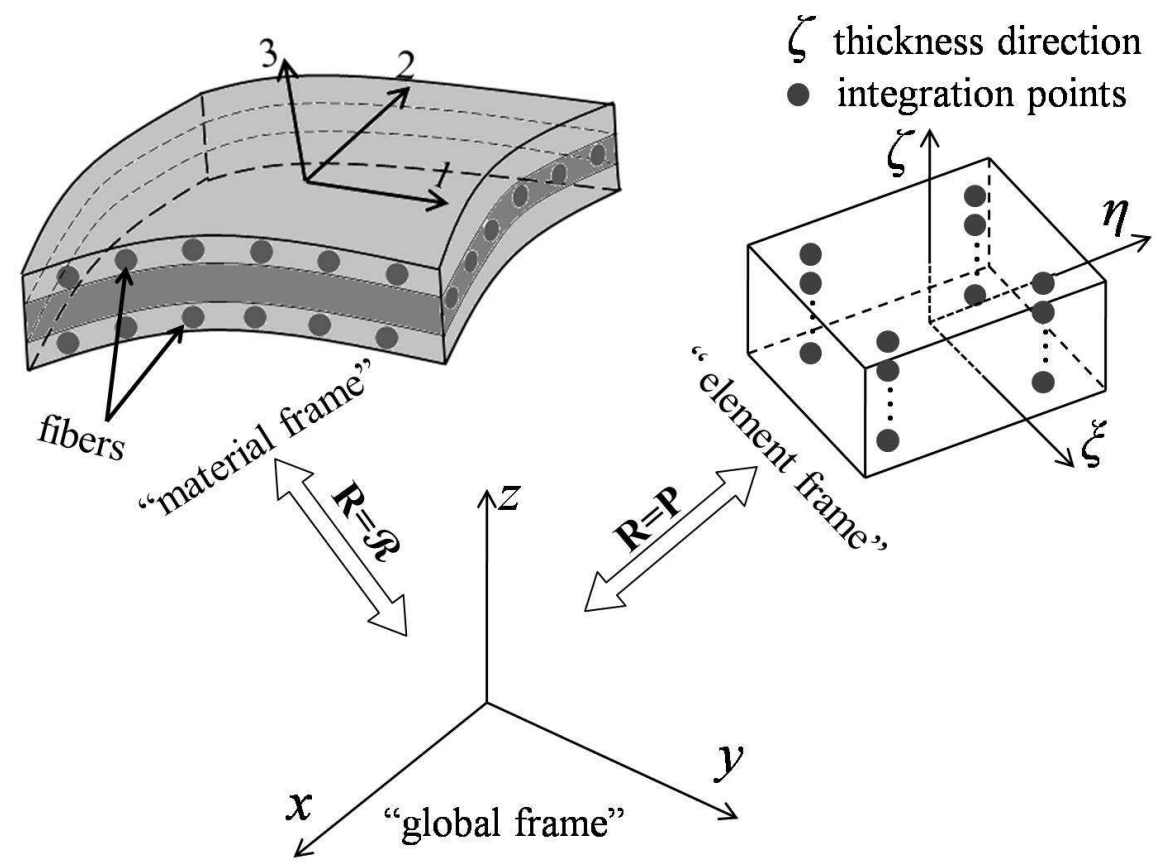

Fig. 2. Illustration of the local frames used in the formulation of the SHB solid-shell elements.

\subsection{Orthotropic elastic tangent modulus}

Unlike traditional shell elements, where the plane-stress assumption is used in their formulation, the proposed solid-shell elements are based on a purely three-dimensional approach, with the consideration of fully three-dimensional constitutive laws. This interesting feature makes the SHB elements very attractive, as they allow 3D modeling of thin structures using only a single element layer. In this work, the quadratic SHB elements are formulated within the framework of large strains and coupled with orthotropic elastic behavior, which allows extending their application range to the 3D nonlinear analysis of composite laminates.

The rate form of the stress-strain relationship can be expressed by the following hypoelastic law, defined in the material frame:

$\dot{\boldsymbol{\sigma}}=\mathbf{C}(\theta): \mathbf{D}^{e}$, 
where $\mathbf{D}^{e}$ is the elastic strain rate tensor. The fourth-order orthotropic elasticity tensor $\mathbf{C}(\theta)$ is specified with respect to the local element frame, and can be expressed as follows:

$$
\mathbf{C}(\theta)=\mathbf{T}(\theta) \mathbf{H}^{-1} \mathbf{T}(\theta)^{T},
$$

where the fourth-order tensor $\mathbf{H}$ represents the elastic compliance of the orthotropic material, which is defined in the fiber reference axes $\left(\mathbf{m}_{1}, \mathbf{m}_{2}, \mathbf{m}_{3}\right)$, with $\mathbf{m}_{1}$ being the fiber direction, $\mathbf{m}_{2}$ the transverse direction, and $\mathbf{m}_{3}$ the normal to the ply (see the illustrative Fig. 3). Its expression depends on the engineering constants of the ply and has the following form:

$$
\mathbf{H}=\left[\begin{array}{cccccc}
\frac{1}{E_{1}} & -\frac{v_{21}}{E_{2}} & -\frac{v_{31}}{E_{3}} & 0 & 0 & 0 \\
-\frac{v_{12}}{E_{1}} & \frac{1}{E_{2}} & -\frac{v_{32}}{E_{3}} & 0 & 0 & 0 \\
-\frac{v_{13}}{E_{1}} & -\frac{v_{23}}{E_{2}} & \frac{1}{E_{3}} & 0 & 0 & 0 \\
0 & 0 & 0 & \frac{1}{G_{12}} & 0 & 0 \\
0 & 0 & 0 & 0 & \frac{1}{G_{23}} & 0 \\
0 & 0 & 0 & 0 & 0 & \frac{1}{G_{13}}
\end{array}\right],
$$

where $E_{i}$ represents the Young modulus in the $i$-th direction, $v_{i j}$ is the Poisson ratio associated with the $i$-th and $j$-th directions, and $G_{12}, G_{13}$ and $G_{23}$ are the shear moduli. Furthermore, the thermodynamic constraint on the elastic constants for orthotropic materials, which is expressed by the relation $v_{i j} E_{j}=v_{j i} E_{i}$, should always be satisfied.

The rotation matrix $\mathbf{T}(\theta)$ in Eq. (14), which allows mapping the orthotropic elasticity tensor from the fiber reference axes to the local element frame, takes the following form: 
$\mathbf{T}(\theta)=\left[\begin{array}{cccccc}c^{2} & s^{2} & 0 & 2 c s & 0 & 0 \\ s^{2} & c^{2} & 0 & -2 c s & 0 & 0 \\ 0 & 0 & 1 & 0 & 0 & 0 \\ -c s & c s & 0 & c^{2}-s^{2} & 0 & 0 \\ 0 & 0 & 0 & 0 & -s & s \\ 0 & 0 & 0 & 0 & -s & c\end{array}\right]$,

with $c=\cos (\theta)$ and $s=\sin (\theta)$.

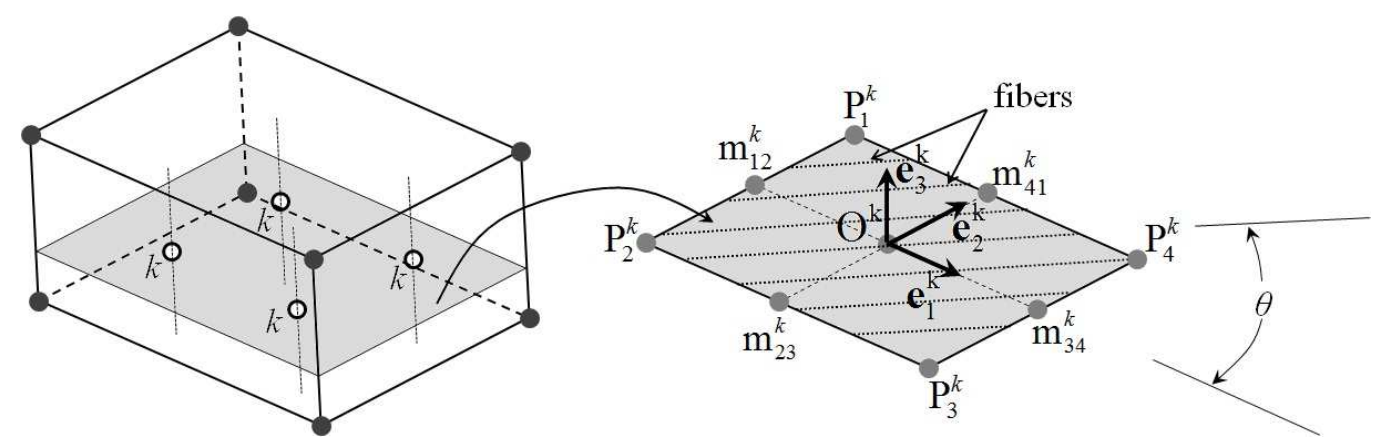

(a) SHB20 hexahedral element

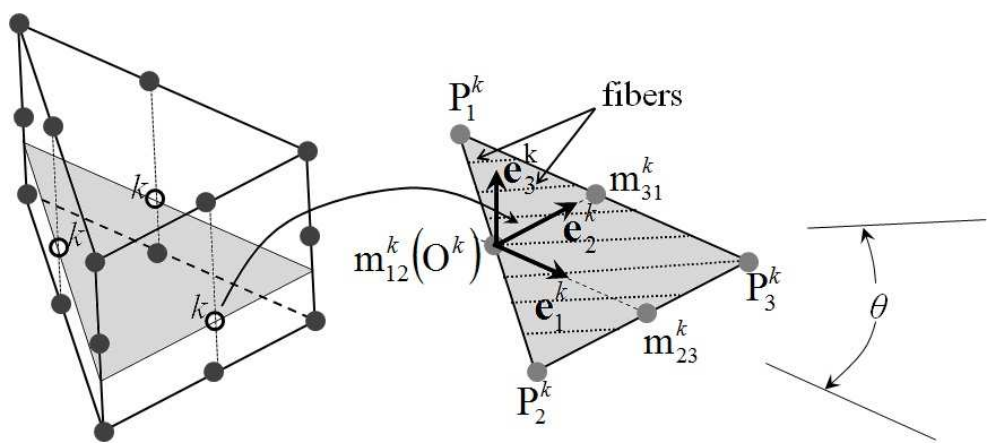

(b) SHB15 prismatic element

Fig. 3. Schematic representation of the fiber orientations with respect to the local element frame.

Several techniques can be adopted for the modeling of multilayered composite structures using solid-shell elements. The simplest one consists in discretizing the entire thickness with one element layer per ply, which leads in the end to as many element layers as plies. However, this technique leads to a large number of degrees of freedom for the composite structure in the case of refined in-plane meshes, which in turn significantly increases the computational time. Another technique can be used, which is based on stacking the different composite plies within a single solid-shell element. In this latter case, each ply is modeled using one integration point and, 
therefore, the stiffness matrix associated with the entire thickness is calculated using the classical Gauss integration scheme over all integration points. This alternative technique, which is adopted in this work, allows for 3D modeling of multilayered composite structures using only a single solid-shell element through the thickness.

The numerical implementation of this technique is described below. At each integration point of a given element, the local element frame, which is defined by its unit directions $\left(\mathbf{e}_{1}^{k}, \mathbf{e}_{2}^{k}, \mathbf{e}_{3}^{k}\right)$, is computed using the coordinates of the element nodes, as illustrated in Fig. 3 for the SHB20 and SHB15 elements. Once the element frame is built, the orthotropic elasticity tensor is calculated for each ply (i.e., at each integration point) in the local element frame using Eq. (14). Then, this operation is repeated for all plies of the composite laminate, as shown in Fig. 4, which allows considering several stacking sequences of plies within a single element layer.

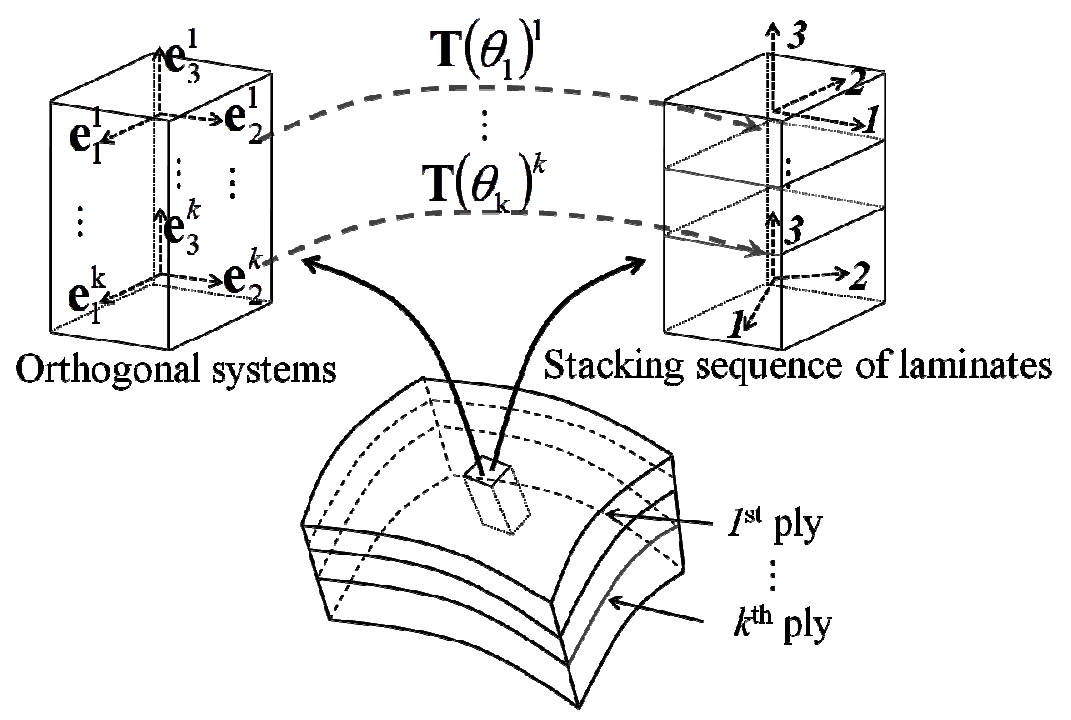

Fig. 4. Illustration of the stacking sequence technique for the multilayered composite material.

\section{Benchmarks problems and simulation results}

The proposed quadratic SHB elements have been implemented into the finite element code ABAQUS/Standard via User-Element (UEL) subroutines. In this section, a representative set of numerical benchmark tests is chosen to assess their performance in the context of composite structure applications. All finite element models of the subsequent tests are discretized using the following nomenclature: for hexahedral elements, meshes of $\mathrm{N}_{1} \times \mathrm{N}_{2} \times \mathrm{N}_{3}$ elements are adopted, 
where $\mathrm{N}_{1}$ denotes the number of elements in the length direction, $\mathrm{N}_{2}$ is the number of elements in the width direction, and $\mathrm{N}_{3}$ is the number of elements in the thickness direction; while for meshes with prismatic elements, the nomenclature adopted is $\left(\mathrm{N}_{1} \times \mathrm{N}_{2} \times 2\right) \times \mathrm{N}_{3}$, which corresponds to twice the total number of elements involved in hexahedron-based meshes, due to the subdivision of each hexahedron into two prisms. Since only a single SHB element layer is used to model the entire thickness of composite laminates, the parameter $\mathrm{N}_{3}$ is set equal to one for all simulations.

In order to demonstrate the capabilities of the quadratic SHB elements, all simulation results are compared, on the one hand, to reference solutions taken from the literature and, on the other hand, to the results provided by ABAQUS quadratic solid elements as well as ABAQUS linear solid-shell elements, using the same in-plane meshes. Note that no quadratic solid-shell counterparts exist in the ABAQUS software. The description of all finite elements involved in this paper is given in Table 1. It is worth noting that the quadratic hexahedral ABAQUS element (C3D20) is able to model composite laminates with only a single element layer through the thickness, which corresponds to the same strategy used for the proposed SHB elements. However, this is not the case for the quadratic prismatic ABAQUS element (C3D15). Because the latter is not able to model composite laminates with only a single element layer through the thickness, one has to resort to the alternative strategy, which consists in using as many C3D15 element layers as plies. Note also that, in the following simulations, the obtained numerical results that are achieved with the designed meshes correspond to the converged solutions. Moreover, the total number of increments and associated number of iterations, NINC and NITER, respectively, required to obtain the converged solutions are reported at the end of the simulations, which allows evaluating the efficiency and convergence of the proposed SHB elements with respect to their ABAQUS counterparts. To achieve this, the default automatic incrementation procedure in ABAQUS is adopted for both the SHB and ABAQUS elements, with initial and maximum time steps equal to 0.1 and 1 , respectively. 


\section{Table 1}

Prismatic and hexahedral finite elements used in the simulations.

\begin{tabular}{ccl}
\hline & SHB15 & $\begin{array}{l}\text { 15-node prismatic solid-shell element with a user-defined number of } \\
\text { through-thickness integration points }\end{array}$ \\
\cline { 2 - 3 } $\begin{array}{c}\text { Prismatic } \\
\text { elements }\end{array}$ & C3D15 & $\begin{array}{l}\text { 15-node prismatic solid element with three integration points through } \\
\text { the thickness }\end{array}$ \\
\cline { 2 - 3 } & SC6R & $\begin{array}{l}\text { 6-node prismatic solid-shell element with a user-defined number of } \\
\text { through-thickness integration points }\end{array}$ \\
\cline { 2 - 3 } $\begin{array}{c}\text { Hexahedral } \\
\text { elements }\end{array}$ & SHB20 & $\begin{array}{l}\text { 20-node hexahedral solid-shell element with a user-defined number } \\
\text { of through-thickness integration points }\end{array}$ \\
\cline { 2 - 3 } & SC8R & $\begin{array}{l}\text { 20-node hexahedral solid element with three integration points } \\
\text { through the thickness }\end{array}$ \\
\cline { 2 - 3 } & $\begin{array}{l}\text { 8-node hexahedral solid-shell element with a user-defined number of } \\
\text { through-thickness integration points }\end{array}$ \\
\hline
\end{tabular}

\subsection{Cantilever plate with ply dropoffs}

It is well known that traditional shell elements are widely used in the literature for the simulation of composite structures due to their high efficiency and accuracy. However, they are not able to model composite laminates with zones having variable thickness, which is the case of the present nonlinear cantilever plate test with ply dropoffs. Therefore, only continuum finite elements, such as the proposed solid-shell ones, are capable of modeling this type of composite structures that require a three-dimensional representation of the geometry.

Fig. 5 illustrates the geometry of the composite cantilever plate, with three ply dropoffs. Each of these three ply dropoffs is modeled with a single finite element layer, and contains two plies with fiber directions oriented at $-45^{\circ} / 45^{\circ}$ from the bottom to the top with respect to the length direction. Hence, the thick end (clamped end) contains six plies, while the thinnest end (free end) contains two plies. The cantilever plate is subjected to a bending force $\mathrm{F}=6 \times 10^{9} \mathrm{~h}^{3}$, with $\mathrm{h}$ being the thickness of each ply (all plies have the same thickness). The engineering constants of the orthotropic material are $E_{11}=2.5 \times 10^{10}, E_{22}=E_{33}=10^{9}, v_{12}=v_{13}=v_{23}=0.2$, and $G_{12}=G_{13}=G_{23}=0.5 \times 10^{9}$. The length of the plate is $\mathrm{L}=12$, and the width is $\mathrm{b}=6$, with the top 
layer removed after successive dropoffs at the coordinates $x=4$ and $x=8$ in the length direction. Three different values for the ply thickness are considered, i.e., $\mathrm{h}=0.1,0.01$, and 0.004 .

For the hexahedral elements (i.e., SC8R, SHB20 and C3D20), the plate is discretized using six elements in the length direction and three elements in the width direction. In the case of the prismatic elements (i.e., SC6R, SHB15 and C3D15), 24 elements in the length direction and six elements in the width are used. The obtained final deflections, normalized with respect to the reference solutions given in [25], as well as the required NINCs and NITERs are reported in Table 2 and Table 3, respectively, for the three ply thicknesses considered. One can observe that, in the case of ply thickness $\mathrm{h}=0.1$, both $\mathrm{SHB}$ and ABAQUS elements predict well the maximum deflection of this composite cantilever plate, with equivalent NINC and NITER (see Table 3). However, for smaller thicknesses of plies (i.e., large length to thickness ratios $\mathrm{L} / \mathrm{h}$ ), the quadratic SHB elements as well as the ABAQUS solid-shell elements provide the best predictions with very high accuracy, while the quadratic ABAQUS solid elements reveal their limitations in modeling very thin 3D structures.

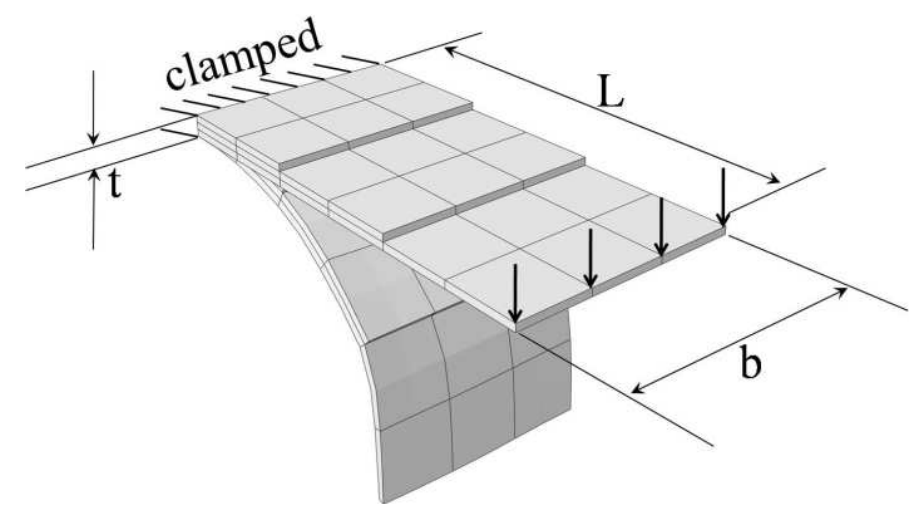

Fig. 5. Cantilever plate with ply dropoffs. 
Table 2

Normalized deflections for the cantilever plate with ply dropoffs.

\begin{tabular}{cccccccc}
\hline \multirow{2}{*}{ Ply thickness } & $\begin{array}{c}\text { aspect ratio } \\
\text { L/h }\end{array}$ & SC6R & C3D15 & SHB15 & SC8R & C3D20 & SHB20 \\
& 120 & 1.0684 & 1.0111 & 0.9954 & 1.1258 & 1.0003 & 0.9950 \\
0.1 & 1200 & 0.9865 & 0.9317 & 0.9980 & 0.9898 & 0.6732 & 1.0061 \\
0.01 & 3000 & 0.9851 & 0.8338 & 1.0086 & 0.9985 & 0.4644 & 1.0099 \\
\hline
\end{tabular}

Table 3

NINC and NITER required by the SHB and ABAQUS elements for the cantilever plate with ply dropoffs.

\begin{tabular}{cccccccc}
\hline Ply thickness & Element & SC6R & C3D15 & SHB15 & SC8R & C3D20 & SHB20 \\
\hline \multirow{2}{*}{0.1} & NINC & 16 & 14 & 17 & 20 & 14 & 17 \\
& NITER & 74 & 64 & 93 & 92 & 62 & 94 \\
\hline \multirow{2}{*}{0.01} & NINC & 47 & 24 & 40 & 46 & 15 & 43 \\
& NITER & 203 & 92 & 200 & 207 & 73 & 214 \\
\hline \multirow{2}{*}{0.004} & NINC & 67 & 21 & 57 & 74 & 14 & 60 \\
& NITER & 292 & 103 & 264 & 280 & 68 & 295 \\
\hline
\end{tabular}

\subsection{Cantilever bending of a laminated beam}

As illustrated in Fig. 6, a cantilever laminated beam, which is subjected to a bending load at its free end, is analyzed in this section. Four different stacking sequences with respect to the length direction are considered for the laminated beam, i.e., $\left[0^{\circ} / 90^{\circ} / 0^{\circ}\right],\left[30^{\circ} /-60^{\circ} /-60^{\circ} / 30^{\circ}\right],\left[-45^{\circ} / 45^{\circ} /-\right.$ $\left.45^{\circ} / 45^{\circ}\right]$ and $\left[90^{\circ} / 0^{\circ} / 90^{\circ}\right]$. The engineering constants of the orthotropic material are $E_{11}=10^{6}$, $E_{22}=E_{33}=0.3 \times 10^{6}, v_{12}=v_{13}=v_{23}=0.25, G_{12}=G_{13}=0.15 \times 10^{6}, G_{23}=0.12 \times 10^{6}$. 


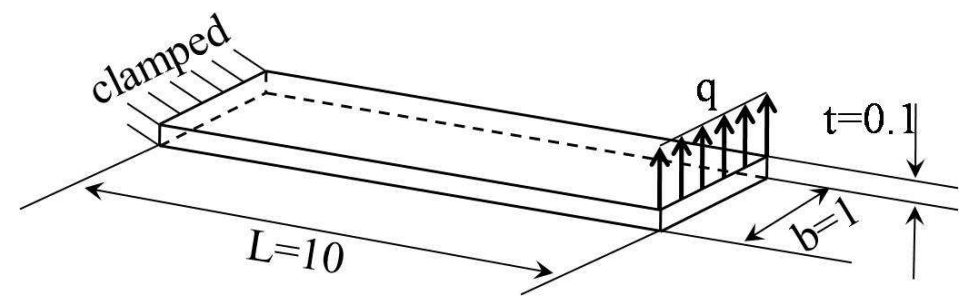

Fig. 6. Cantilever laminated beam subjected to a bending load.

Fig. 7 depicts the load-deflection curves obtained with the SHB and ABAQUS elements, along with the reference solutions taken from [17]. The NINC and NITER required by all elements are reported in Table 4. Overall, one can observe that both the proposed quadratic SHB elements and ABAQUS elements show excellent agreement with the reference solutions corresponding to the four stacking sequences. Moreover, the NINC and NITER required by the SHB elements are relatively equivalent to those involved by ABAQUS elements, which reveals the good convergence of the proposed elements.

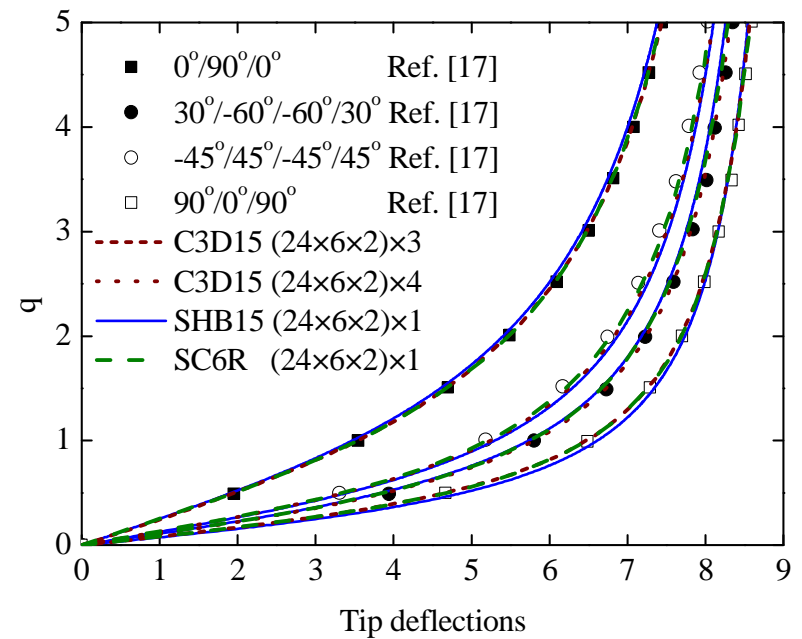

(a) prismatic elements

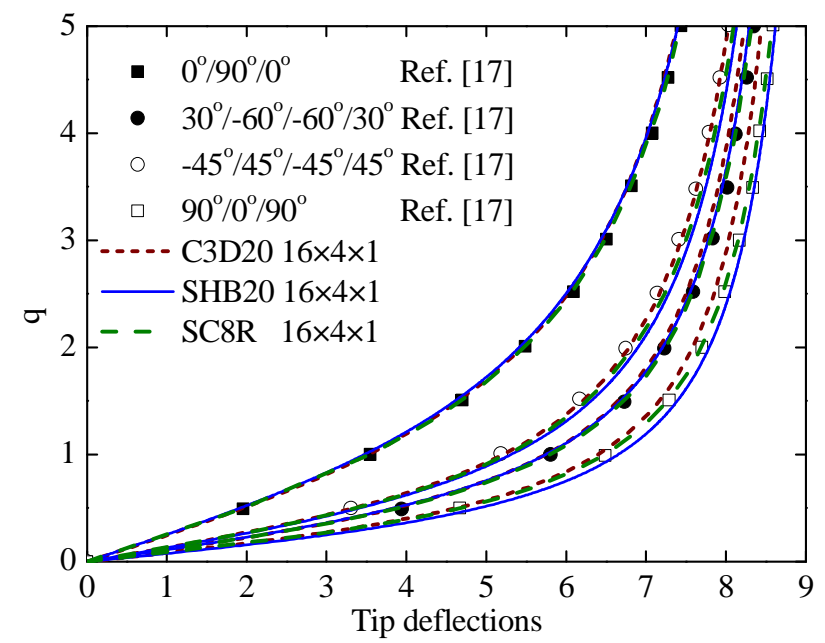

(b) hexahedral elements

Fig. 7. Load-deflection curves for the cantilever laminated beam. 


\section{Table 4}

NINC and NITER required by the SHB and ABAQUS elements for the cantilever laminated beam.

\begin{tabular}{cccccccc}
\hline Stacking sequence & Element & SC6R & C3D15 & SHB15 & SC8R & C3D20 & SHB20 \\
\hline \multirow{2}{*}[0^{\circ}/90^{\circ}/0^{\circ}]{} & NINC & 17 & 9 & 18 & 16 & 13 & 18 \\
& NITER & 82 & 50 & 86 & 80 & 63 & 88 \\
\hline \multirow{2}{*}[30^{\circ}/-60^{\circ}/-60^{\circ}/30^{\circ}]{} & NINC & 21 & 16 & 23 & 21 & 17 & 24 \\
& NITER & 111 & 83 & 119 & 110 & 90 & 127 \\
\hline \multirow{2}{*}[-45^{\circ}/45^{\circ}/-45^{\circ}/45^{\circ}]{} & NINC & 21 & 12 & 24 & 22 & 17 & 21 \\
& NITER & 108 & 80 & 118 & 101 & 92 & 109 \\
\hline \multirow{2}{*}[90^{\circ}/0^{\circ}/90^{\circ}]{} & NINC & 23 & 17 & 27 & 26 & 22 & 30 \\
& NITER & 104 & 95 & 138 & 122 & 115 & 147 \\
\hline
\end{tabular}

\subsection{Clamped laminated square plate under uniform pressure}

In this nonlinear benchmark problem, a fully clamped laminated square plate is subjected to a uniformly distributed pressure, as illustrated in Fig. 8. The laminated square plate is made of four plies, which are stacked with the following symmetric sequence $\left[0^{\circ} / 90^{\circ} / 90^{\circ} / 0^{\circ}\right]$. The length-tothickness ratio $\mathrm{L} / \mathrm{t}$ of the square plate is set equal to 125 . The engineering constants of the orthotropic material are $E_{11}=1.8282 \times 10^{6}, E_{22}=E_{33}=1.8315 \times 10^{6}, v_{12}=v_{13}=v_{23}=0.23913$, $G_{12}=G_{13}=G_{23}=3.125 \times 10^{5}$. Considering the problem symmetry, only one quarter of the plate is discretized. In order to compare the numerical results with the reference solutions taken from the literature, the predictions are depicted in terms of non-dimensional load-displacement curves, which are based on the dimensionless load parameter $\bar{q}=(L / t)^{4} q / E_{22}$ and the dimensionless displacement $\overline{\mathrm{w}}=\mathrm{w} / \mathrm{t}$, where $\mathrm{q}$ and $\mathrm{w}$ are the uniform pressure and the out-of-plane displacement at the central point of the square plate, respectively. Fig. 9 shows the simulation results obtained with the SHB and ABAQUS elements, along with the reference solutions taken from references [37] and [38], while the required NINC and NITER are listed in Table 5. It can be seen that the proposed SHB elements perform very well with respect to the reference solutions, which is also the case for the ABAQUS elements, although the C3D15 element requires more finite elements in the mesh, thus involving overall a larger number of degrees of freedom. 
Moreover, the predicted deflections are achieved with equivalent NINC and NITER for all investigated elements, which proves again the good convergence of the proposed SHB elements.

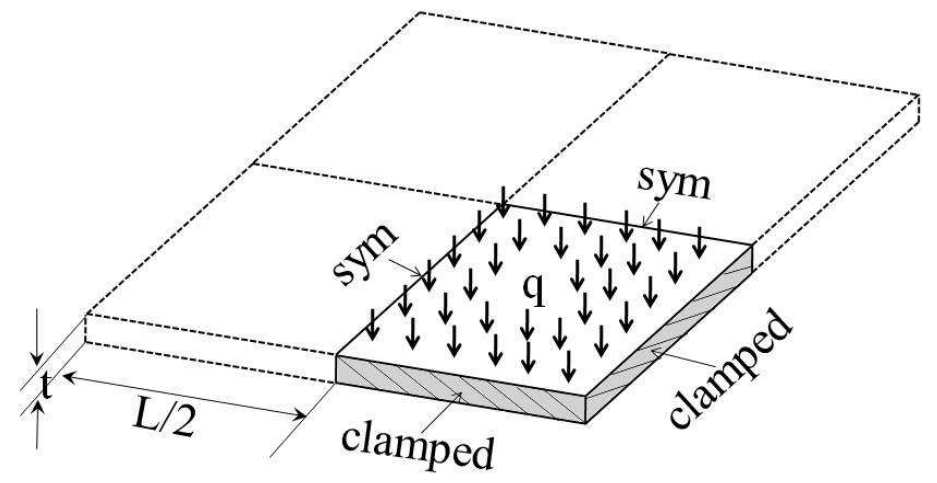

Fig. 8. Clamped laminated square plate subjected to a uniform pressure.

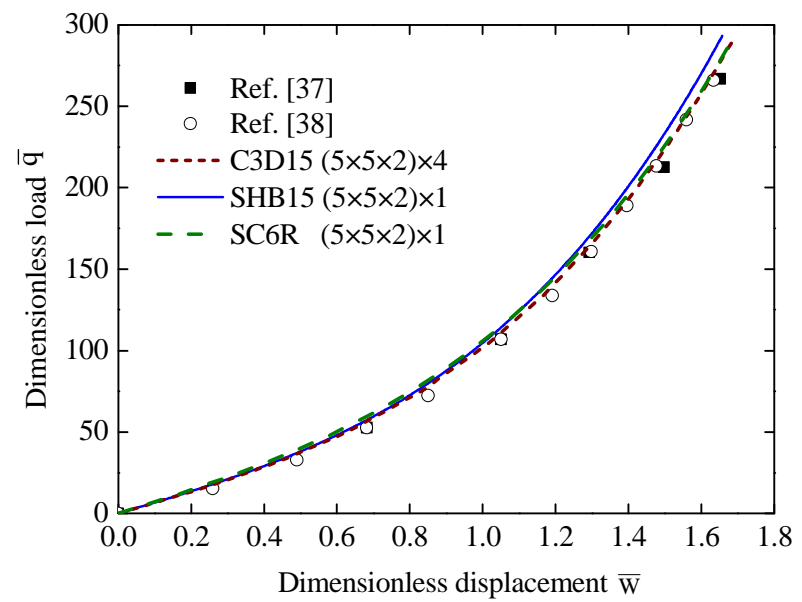

(a) prismatic elements

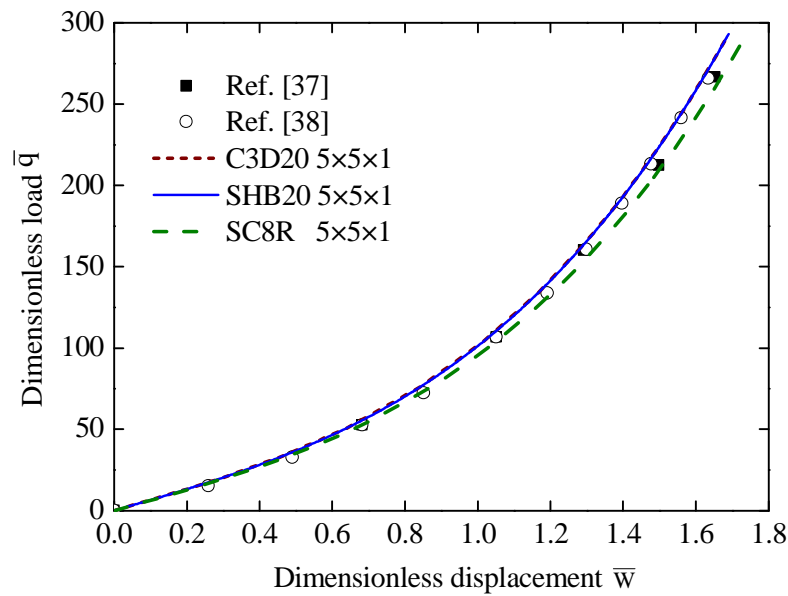

(b) hexahedral elements

Fig. 9. Non-dimensional load-displacement curves at the center point for the laminated square plate.

Table 5

NINC and NITER required by the SHB and ABAQUS elements for the laminated square plate.

\begin{tabular}{ccccccc}
\hline Element & SC6R & C3D15 & SHB15 & SC8R & C3D20 & SHB20 \\
\hline NINC & 6 & 6 & 6 & 6 & 6 & 6 \\
NITER & 16 & 18 & 18 & 18 & 18 & 20 \\
\hline
\end{tabular}




\subsection{Slit laminated annular plate}

The slit annular plate problem is considered in the literature as a popular benchmark test for the performance assessment of finite elements in the framework of large displacements and rotations (see, e.g., [12, 17, 39-42]). This annular plate, as illustrated in Fig. 10, is clamped at one end, while the other free end is subjected to a line shear load P. The inner and outer radii of the annular plate are equal to $R_{i}=6$ and $R_{o}=10$, respectively, while the thickness is $t=0.03$. Two stacking sequences with respect to the radius direction are analyzed here for the composite laminate, i.e., $\left[0^{\circ} / 90^{\circ} / 0^{\circ}\right]$ and $\left[90^{\circ} / 0^{\circ} / 90^{\circ}\right]$. The engineering constants of the orthotropic material are $E_{11}=2 \times 10^{7}, \quad E_{22}=E_{33}=6 \times 10^{6}, \quad v_{12}=v_{13}=0.3, \quad v_{23}=0.25, \quad G_{12}=G_{13}=3 \times 10^{6} ，$ $G_{23}=2.4 \times 10^{6}$. Fig. 11 reports the load-out-of-plane vertical deflection curves at the corner point B of the annular plate, as obtained with the SHB and ABAQUS elements, along with the reference solutions taken from [12, 17], while the corresponding NINC and NITER are summarized in Table 6. It can be seen that the prismatic SHB15 element shows excellent agreement with the reference solutions, which is also the case for the prismatic C3D15 ABAQUS element. For the latter, one recalls that three element layers are required to model the three plies of the laminated annular plate, while only a single layer is used for the proposed SHB elements. Note that a finer mesh is required for the prismatic SC6R ABAQUS solid-shell element in order to achieve an accurate solution. As to the hexahedral elements, Fig. 11 reveals that the proposed SHB20 element provides the closest predictions, with respect to the reference solutions, along the entire loading history, while it requires slightly higher NINC and NITER than ABAQUS elements to obtain accurate solution (see Table 6). 


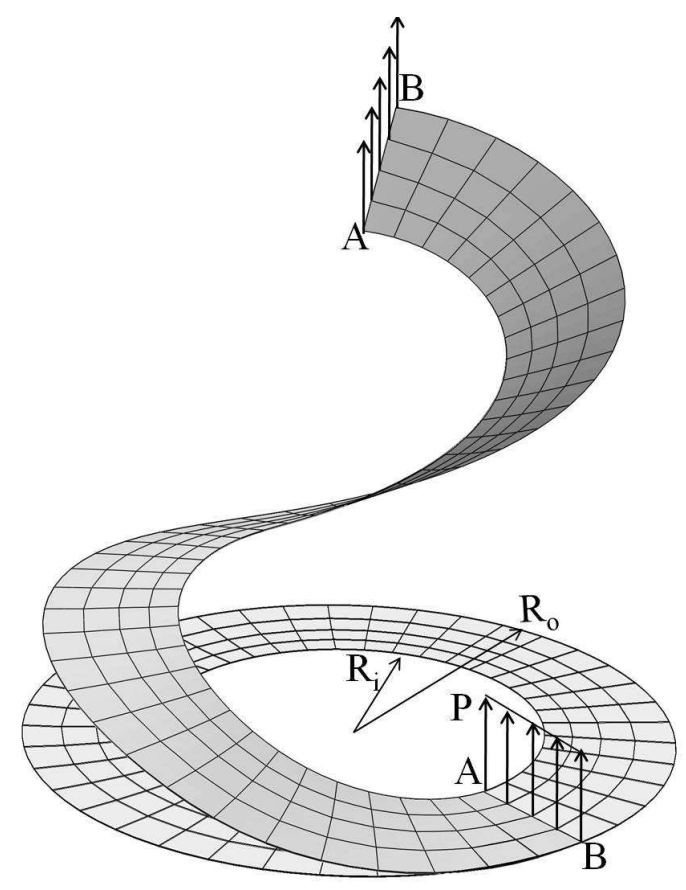

Fig. 10. Undeformed and deformed configurations of the slit laminated annular plate.

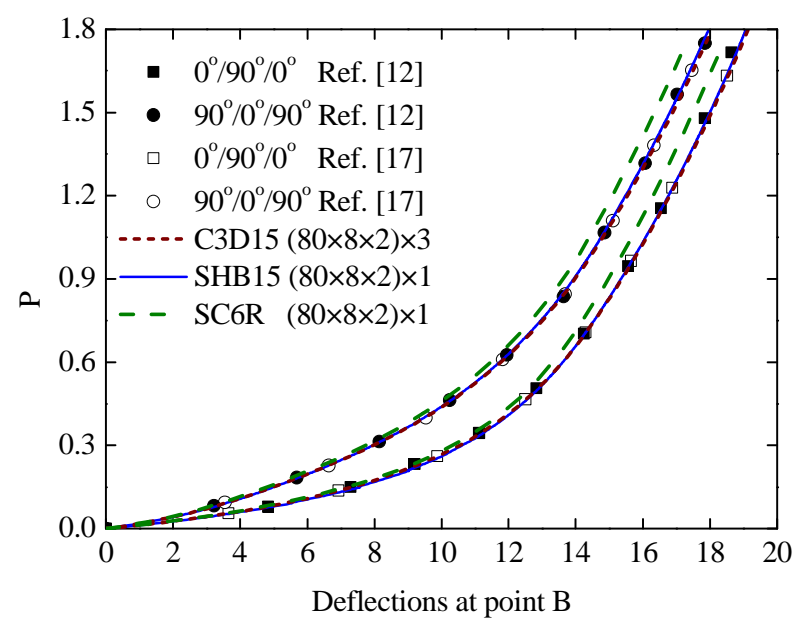

(a) prismatic elements

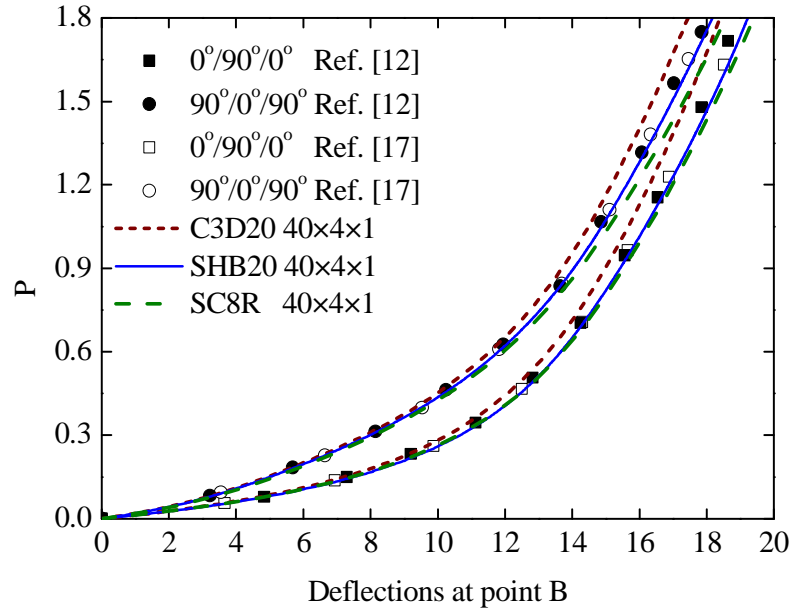

(b) hexahedral elements

Fig. 11. Load-deflection curves at the corner point B for the slit laminated annular plate. 


\section{Table 6}

NINC and NITER required by the SHB and ABAQUS elements for the slit laminated annular plate.

\begin{tabular}{cccccccc}
\hline Stacking sequence & Element & SC6R & C3D15 & SHB15 & SC8R & C3D20 & SHB20 \\
\hline \multirow{2}{*}[0^{\circ}/90^{\circ}/0^{\circ}]{} & NINC & 52 & 29 & 38 & 58 & 29 & 63 \\
& NITER & 261 & 159 & 213 & 260 & 163 & 305 \\
\hline \multirow{2}{*}[90^{\circ}/0^{\circ}/90^{\circ}]{} & NINC & 87 & 36 & 60 & 86 & 38 & 81 \\
& NITER & 409 & 215 & 292 & 390 & 213 & 407 \\
\hline
\end{tabular}

\subsection{Pinched laminated semi-cylindrical shell}

A pinched semi-cylindrical shell, subjected to a concentrated force at its free end, is considered here to assess the performance of the proposed SHB elements in the context of curved composite structures. The geometric dimensions and the boundary conditions of the semicylindrical shell are represented in Fig. 12. Two stacking sequences with respect to the axial direction of the semi-cylinder are considered here for the composite laminate, i.e., $\left[0 \% / 90^{\circ} / 0^{\circ}\right]$ and $\left[90^{\circ} / 0^{\circ} / 90^{\circ}\right]$. The engineering constants of the orthotropic material are $E_{11}=2068.5$, $E_{22}=E_{33}=517.125, v_{12}=v_{13}=v_{23}=0.3, G_{12}=G_{13}=G_{23}=795.6$. Owing to the symmetry, only one half of the model is discretized. Fig. 13 depicts the load-vertical displacement curves at the loading point $\mathrm{A}$, which are obtained using the SHB and ABAQUS elements, along with the reference solutions taken from [42,43], while Table 7 reports the corresponding NINC and NITER. It can be seen that the results obtained with the SHB elements are in excellent agreement with the reference solutions along the entire loading history, while the results given by the prismatic C3D15 ABAQUS element show some discrepancies with respect to the reference solutions at large displacements. As to ABAQUS solid-shell elements (i.e., SC6R and SC8R), the latter provide the farthest results with respect to the reference solution. Note also that the prismatic C3D15 ABAQUS element requires the highest NINC and NITER to achieve the ultimate solution, while comparable convergence is observed for the SHB elements and the hexahedral C3D20 ABAQUS element (see Table 7). 


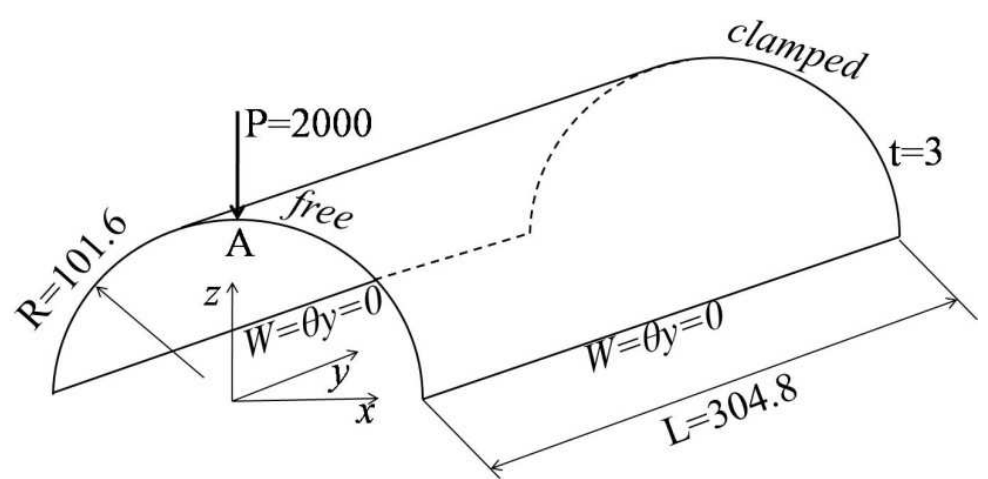

Fig. 12. Pinched laminated semi-cylindrical shell.

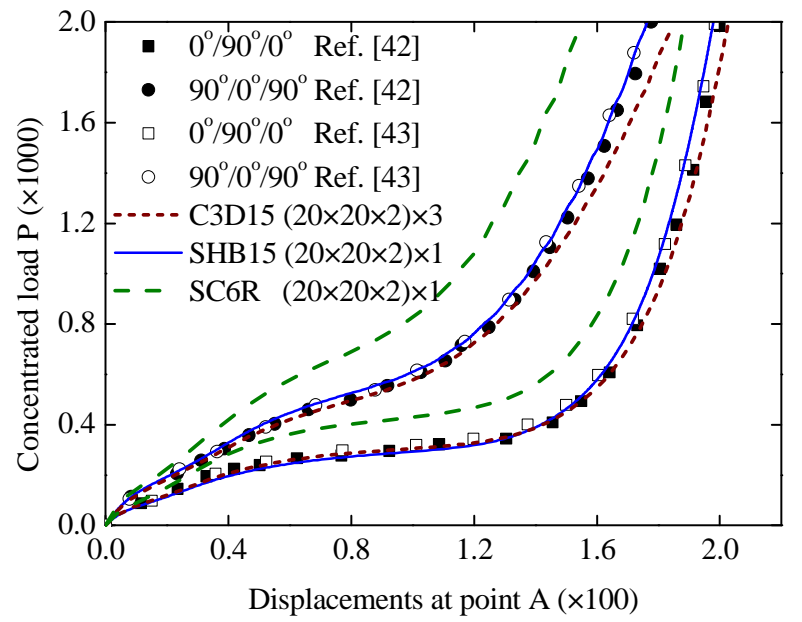

(a) prismatic elements

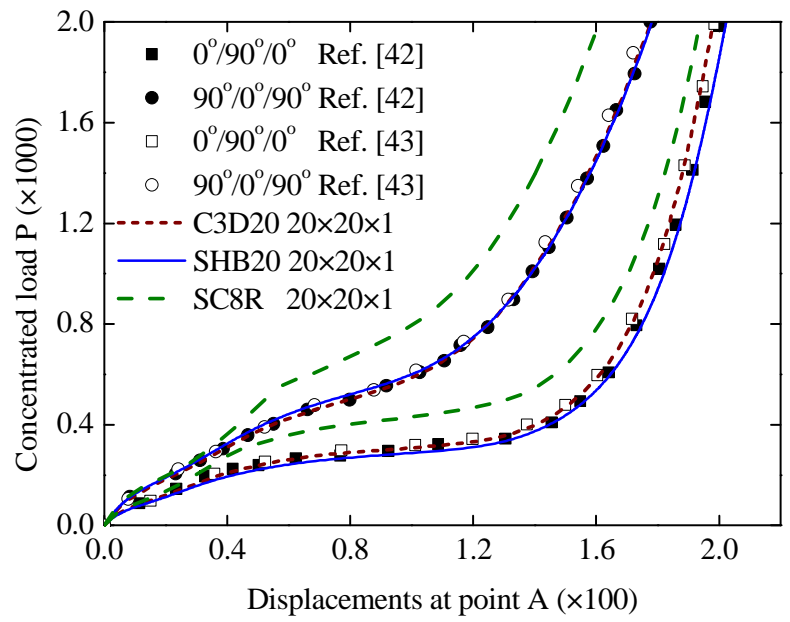

(b) hexahedral elements

Fig. 13. Load-displacement curves for the pinched laminated semi-cylindrical shell.

\section{Table 7}

NINC and NITER required by the SHB and ABAQUS elements for the pinched laminated semicylindrical shell.

\begin{tabular}{cccccccc}
\hline Stacking sequence & Element & SC6R & C3D15 & SHB15 & SC8R & C3D20 & SHB20 \\
\hline$\left[0^{\circ} / 90^{\circ} / 0^{\circ}\right]$ & NINC & 41 & 85 & 38 & 30 & 47 & 47 \\
& NITER & 224 & 496 & 213 & 169 & 287 & 302 \\
\hline \multirow{2}{*}[90^{\circ}/0^{\circ}/90^{\circ}]{} & NINC & 47 & 105 & 60 & 35 & 59 & 51 \\
& NITER & 254 & 594 & 292 & 187 & 326 & 344 \\
\hline
\end{tabular}




\subsection{Pinched laminated hemispherical shell}

Fig. 14 depicts a free laminated hemispherical shell with a $18^{\circ}$ circular hole at its pole. The shell is subjected to a pair of alternating forces at $90^{\circ}$ intervals. The radius and the thickness of this thin hemispherical shell are equal to 10 and 0.08 , respectively. This shell is made of a single ply of fiber-reinforced laminate, with fiber directions aligned to the circumferential direction. The engineering constants of the orthotropic material are (see [44]) $E_{11}=20.46 \times 10^{6}$, $E_{22}=E_{33}=4.092 \times 10^{6}, v_{12}=v_{13}=v_{23}=0.313, G_{12}=G_{13}=2.53704 \times 10^{6}, G_{23}=1.26852 \times 10^{6}$. Considering the problem symmetry, only one quarter of the shell is discretized.

The load-displacement curves at points A and B (see Fig. 14), which are obtained with the SHB and ABAQUS elements, are depicted in Fig. 15 along with the reference solution taken from [44], while Table 8 provides the corresponding NINC and NITER. Note that the final displacements of points $\mathrm{A}$ and $\mathrm{B}$, which are of the same order of magnitude as the shell radius, indicate the large displacements and rotations involved in this test (see also the final deformed shape of the hemispherical shell in Fig. 16). Once again, one can notice the excellent agreement between the results obtained with the proposed SHB elements and the reference solution. Note also that these accurate results are obtained with NINC and NITER that are quite similar to those involved by ABAQUS elements (see Table 8). However, although the adopted meshes correspond to converged results, the simulation results given by ABAQUS elements fall far from the reference solution, showing the limitations of ABAQUS solid-shell and quadratic solid elements in modeling this severe test, which involves strong geometric nonlinearities.

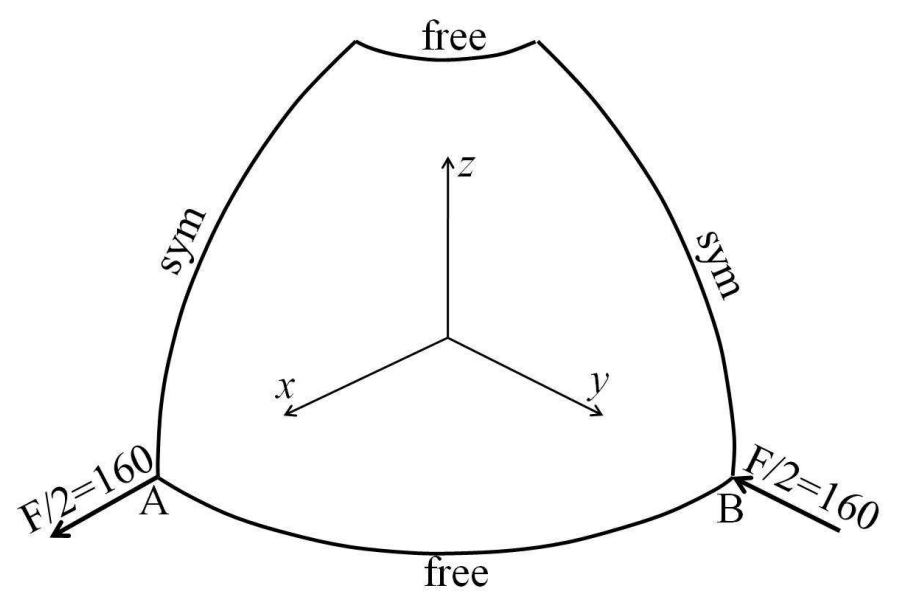

Fig. 14. Pinched laminated hemispherical shell. 


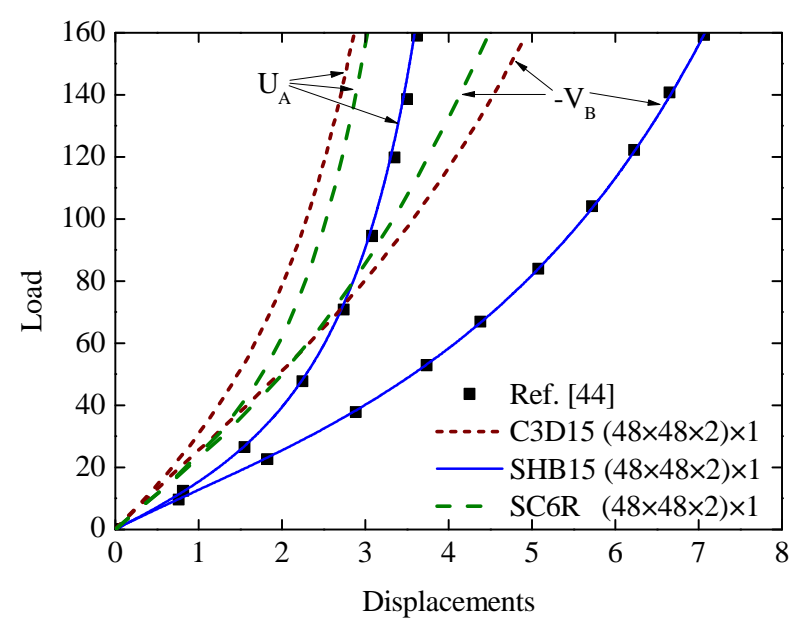

(a) prismatic elements

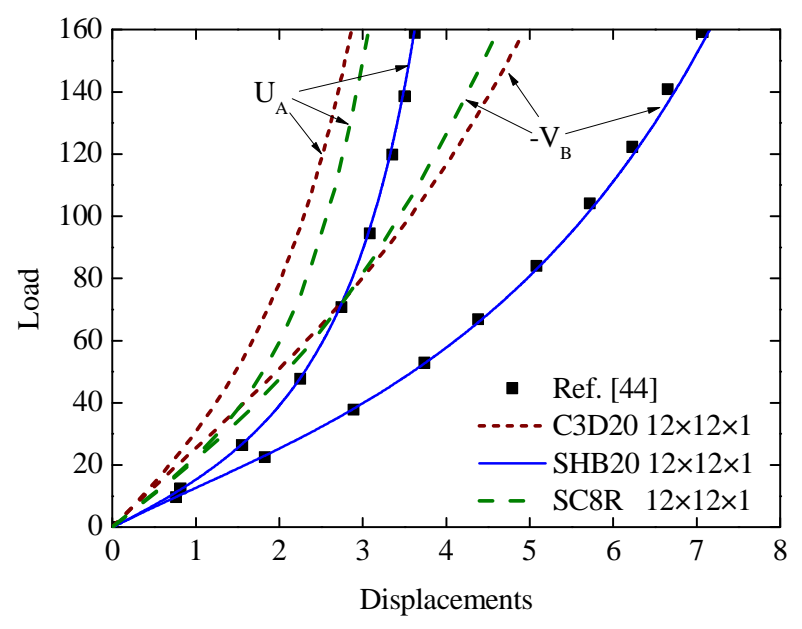

(b) hexahedral elements

Fig. 15. Load-displacement curves at points A and B for the pinched laminated hemispherical shell.

\section{Table 8}

NINC and NITER required by the SHB and ABAQUS elements for the pinched laminated hemispherical shell.

\begin{tabular}{ccccccc}
\hline Element & SC6R & C3D15 & SHB15 & SC8R & C3D20 & SHB20 \\
\hline NINC & 9 & 10 & 10 & 14 & 10 & 10 \\
NITER & 51 & 56 & 73 & 65 & 52 & 75 \\
\hline
\end{tabular}

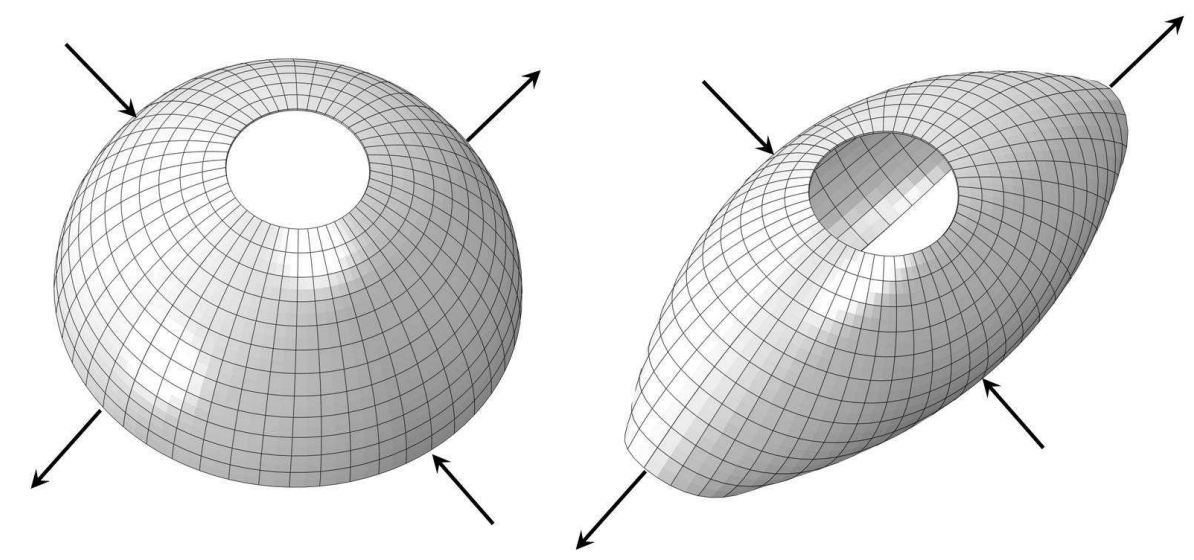

Fig. 16. Undeformed and final deformed shapes of the pinched laminated hemispherical shell. 


\subsection{Hinged laminated cylindrical roof}

Fig. 17 depicts a cylindrical roof with two hinged straight sides (the two other curved sides being free), which is subjected to a concentrated load at its center. Two different thicknesses for this hinged roof are considered, which correspond to a thick roof with $t=12.7$ and a thin roof with $\mathrm{t}=6.35$. Because this nonlinear benchmark test involves geometric-type instabilities (limitpoint buckling), the Riks path-following method together with a fixed arc length is adopted to follow the load-displacement curves beyond the limit points. Consequently, the NINC and NITER required by all finite elements are not shown for this test (see [42]). Four different stacking sequences with respect to the length direction are considered for the composite laminate, i.e., $\left[0^{\circ} / 90^{\circ} / 0^{\circ}\right],\left[90^{\circ} / 0^{\circ} / 90^{\circ}\right],\left[30^{\circ} /-60^{\circ} /-60^{\circ} / 30^{\circ}\right]$ and $\left[-45^{\circ} / 45^{\circ} /-45^{\circ} / 45^{\circ}\right]$. The engineering constants of the orthotropic material are $E_{11}=3300, E_{22}=E_{33}=1100, v_{12}=v_{13}=v_{23}=0.25$, $G_{12}=G_{13}=660, G_{23}=440$. Considering the problem symmetry, only one quarter of the cylindrical roof is discretized for the stacking sequences $\left[0^{\circ} / 90^{\circ} / 0^{\circ}\right]$ and $\left[90^{\circ} / 0^{\circ} / 90^{\circ}\right]$, while a complete model is used in the simulation of the laminates with the stacking sequences $[30 \%-60 \%$ $\left.60^{\circ} / 30^{\circ}\right]$ and $\left[-45^{\circ} / 45^{\circ} /-45^{\circ} / 45^{\circ}\right]$. It is worth noting that two element layers are required to model this cylindrical roof with the ABAQUS linear solid-shell elements (i.e., SC6R and SC8R), since the lateral straight sides are hinged on the mid-surface nodes. By contrast, only a single element layer is used with the quadratic elements, thanks to the availability of mid-surface nodes in these quadratic elements.

The simulation results in terms of load-vertical displacement curves at the central point $\mathrm{A}$ of the thick and thin-hinged cylindrical roofs are reported in Figs. 18-21. From these figures, it can be seen that for the prismatic elements, the results obtained both with the proposed solid-shell element SHB15 and with the ABAQUS C3D15 solid element are in good agreement with the reference solutions taken from [12, 42], while the ABAQUS SC6R solid-shell element provides the farthest results. For the hexahedral elements, however, the proposed SHB20 solid-shell element provides the closest results with respect to the reference solutions, while the simulation results given by the ABAQUS C3D20 solid and SC8R solid-shell elements fall most often far from the reference solutions, thus revealing the limitations of these hexahedral solid and solid-shell elements in modeling this type of limit-point buckling problem (see, e.g., Figs. 18b, 21b-d). To summarize, the snap-through and snap-back phenomena, which are exhibited by the 
present hinged laminated cylindrical roof, are not well captured by the ABAQUS elements (C3D20, SC8R and SC6R), whereas they are well reproduced on the whole by the proposed SHB elements.

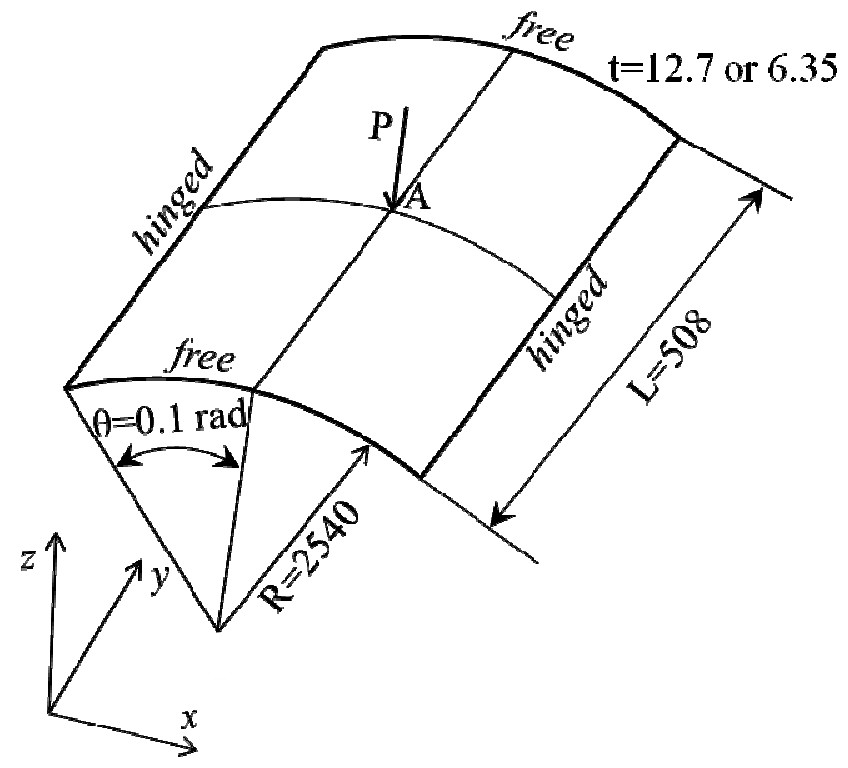

Fig. 17. Hinged laminated cylindrical roof.

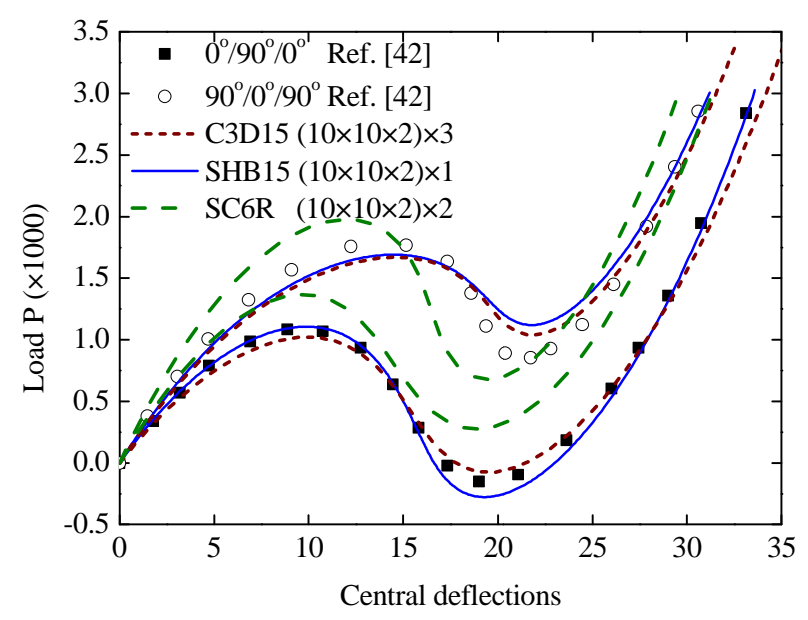

(a) prismatic elements

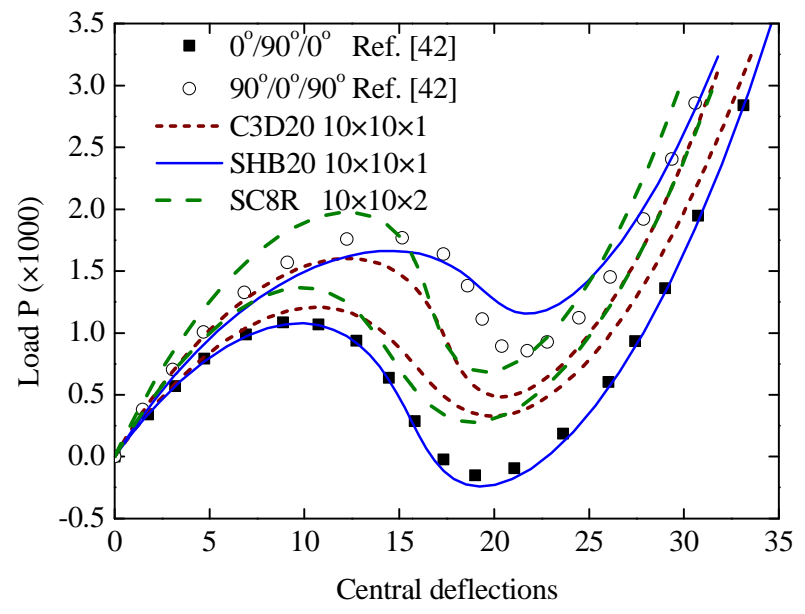

(b) hexahedral elements

Fig. 18. Load-vertical displacement curves at the central point A for the thick laminated cylindrical roof (i.e., $\left[0^{\circ} / 90^{\circ} / 0^{\circ}\right]$ and $\left[90^{\circ} / 0^{\circ} / 90^{\circ}\right]$, with $\left.\mathrm{t}=12.7\right)$. 


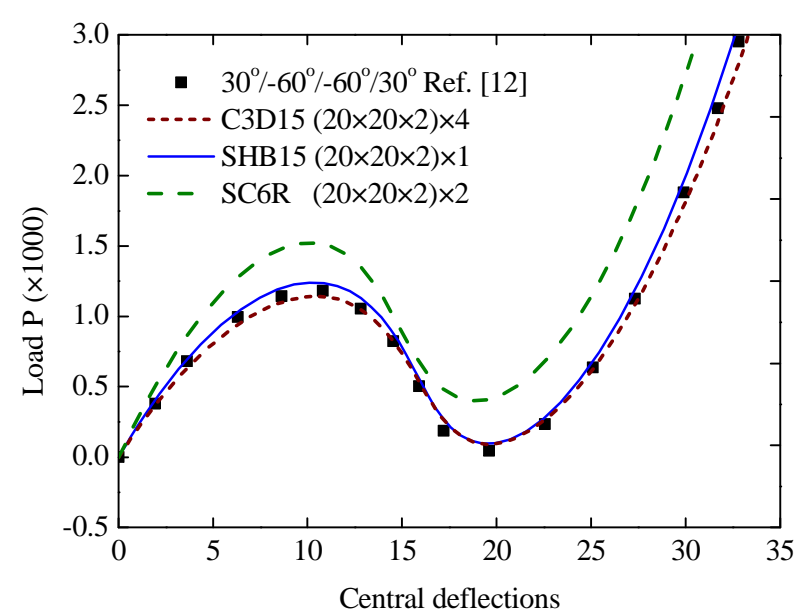

(a) prismatic elements

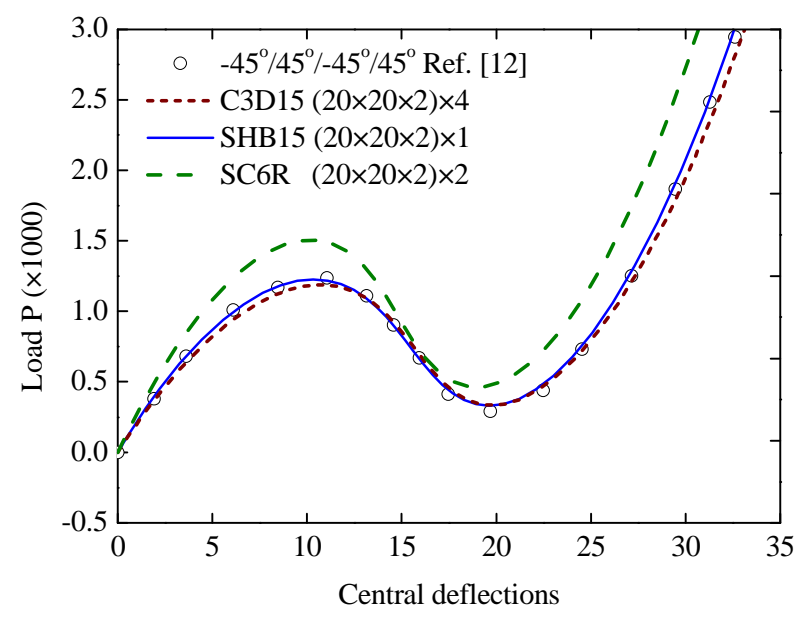

(a) prismatic elements

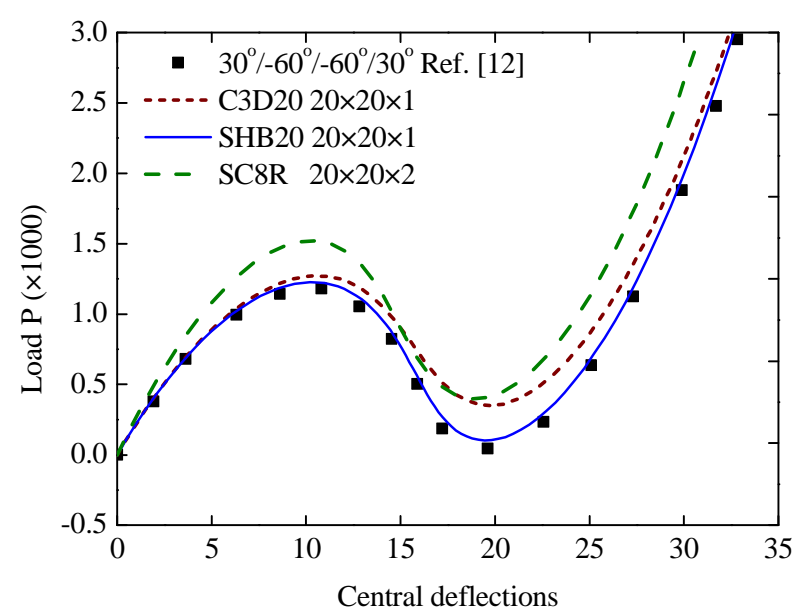

(b) hexahedral elements

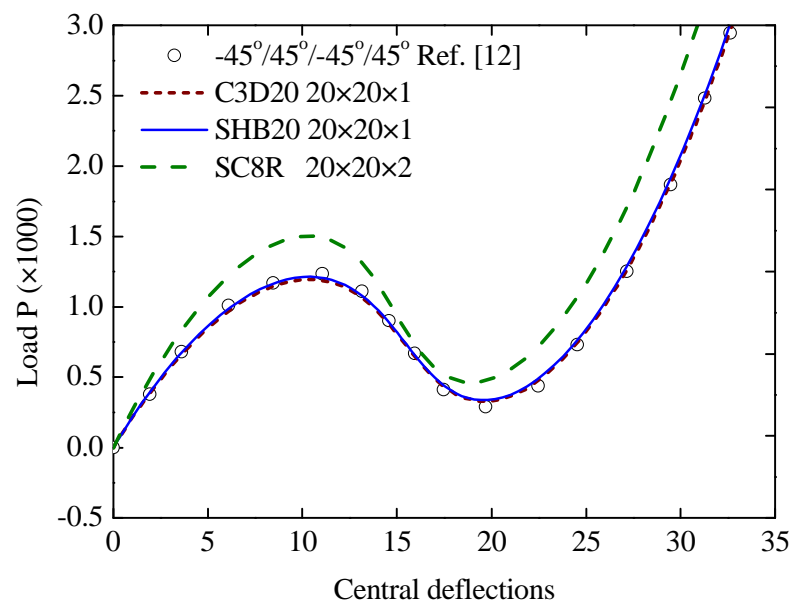

(b) hexahedral elements

Fig. 19. Load-vertical displacement curves at the central point $A$ for the thick laminated cylindrical roof (i.e., $\left[30^{\circ} /-60^{\circ} /-60^{\circ} / 30^{\circ}\right]$ and $\left[-45^{\circ} / 45^{\circ} /-45^{\circ} / 45^{\circ}\right]$, with $\mathrm{t}=12.7$ ). 


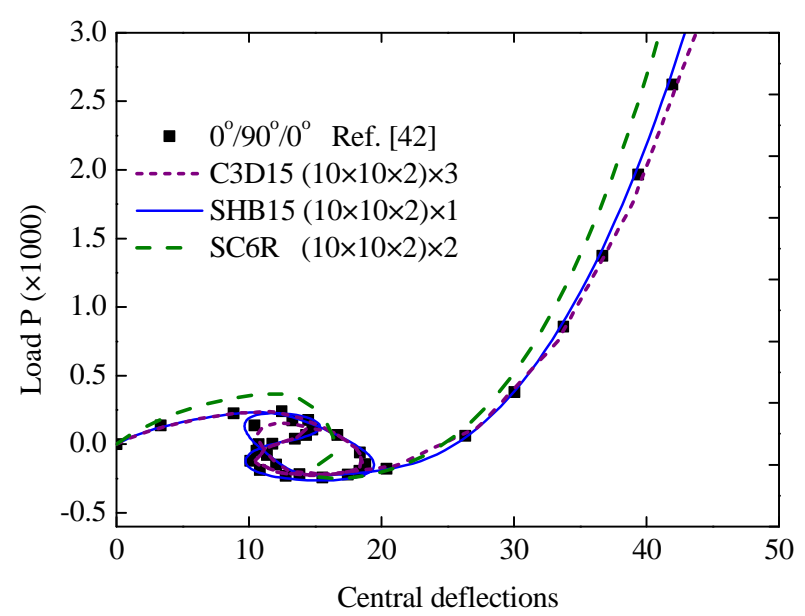

(a) prismatic elements

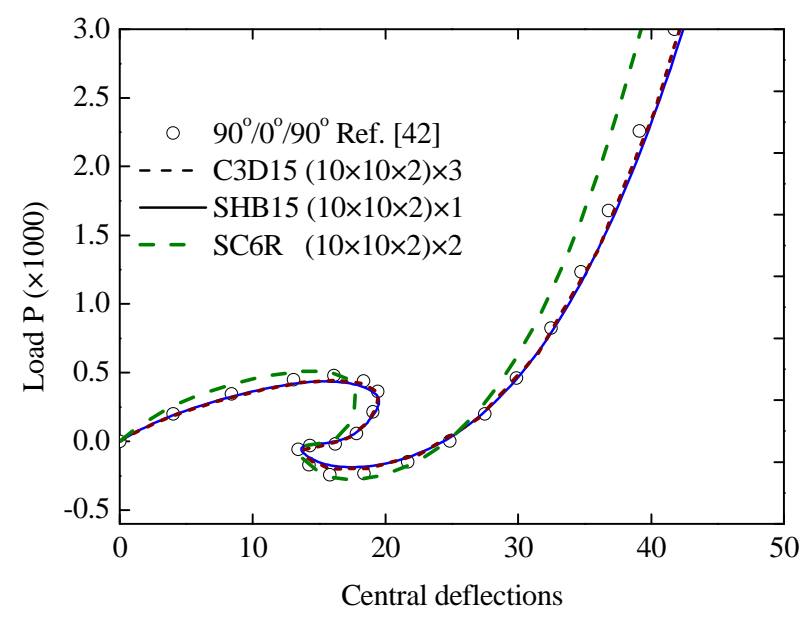

(c) prismatic elements

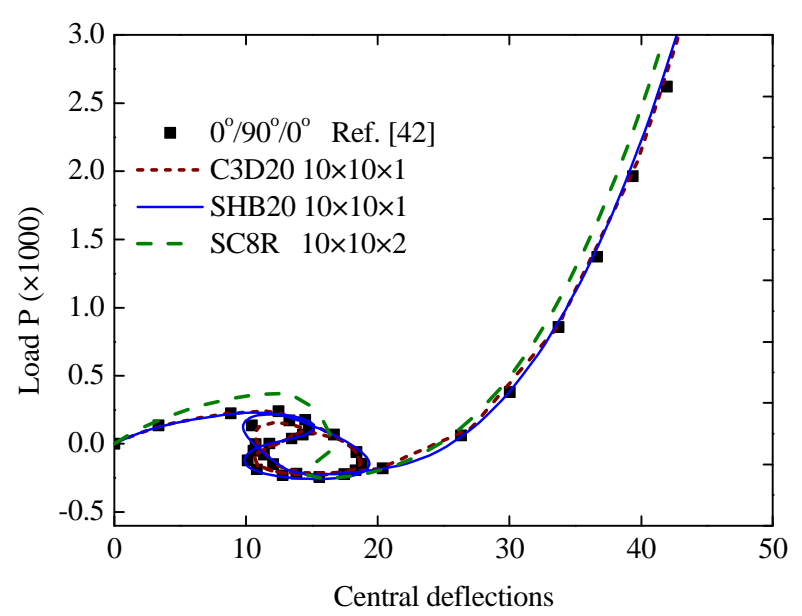

(b) hexahedral elements

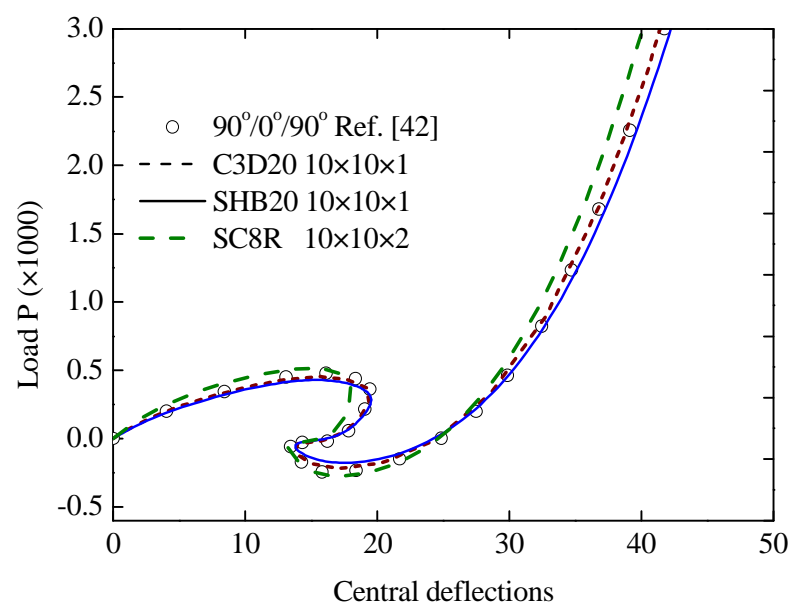

(d) hexahedral elements

Fig. 20. Load-vertical displacement curves at the central point A for the thin laminated cylindrical roof (i.e., $\left[0^{\circ} / 90^{\circ} / 0^{\circ}\right]$ and $\left[90^{\circ} / 0^{\circ} / 90^{\circ}\right]$, with $\mathrm{t}=6.35$ ). 


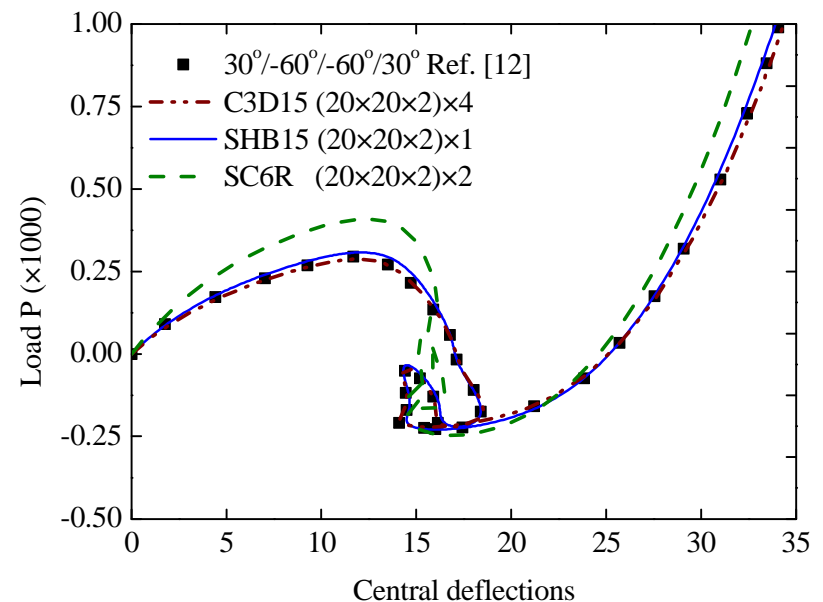

(a) prismatic elements

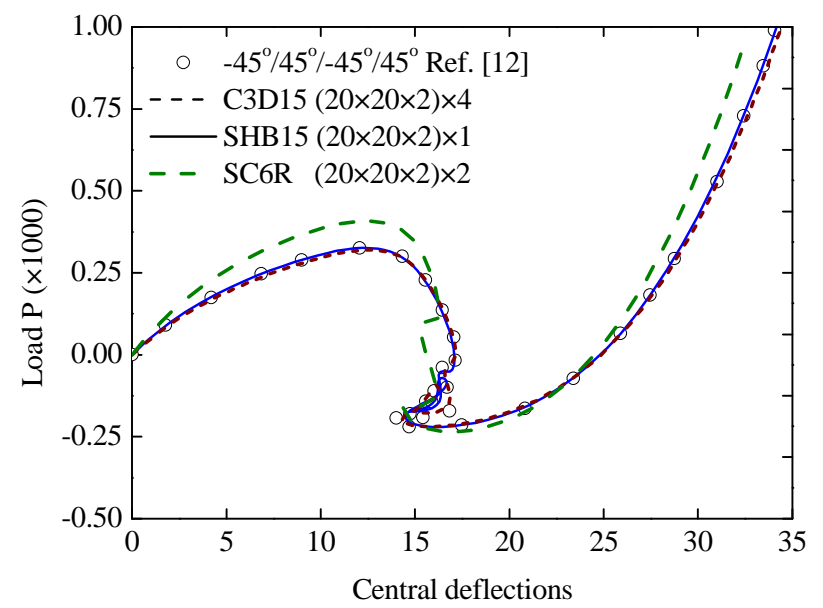

(c) prismatic elements

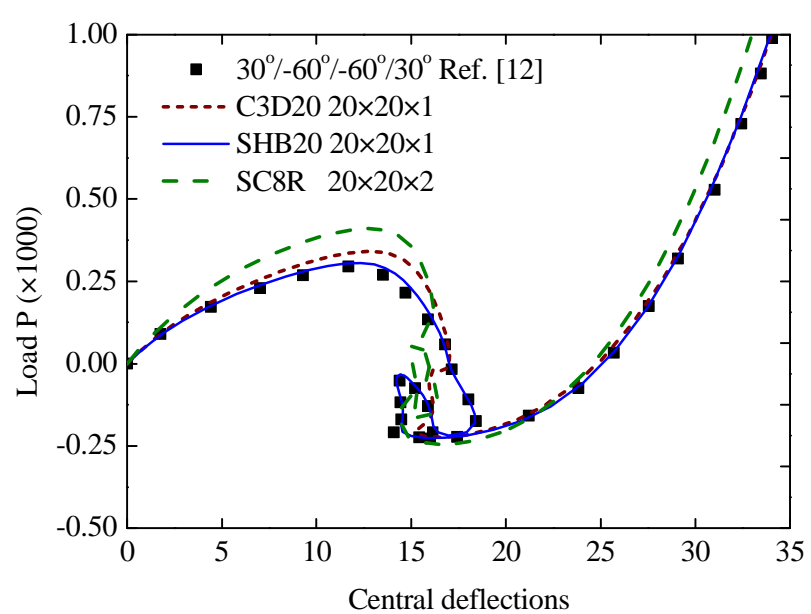

(b) hexahedral elements

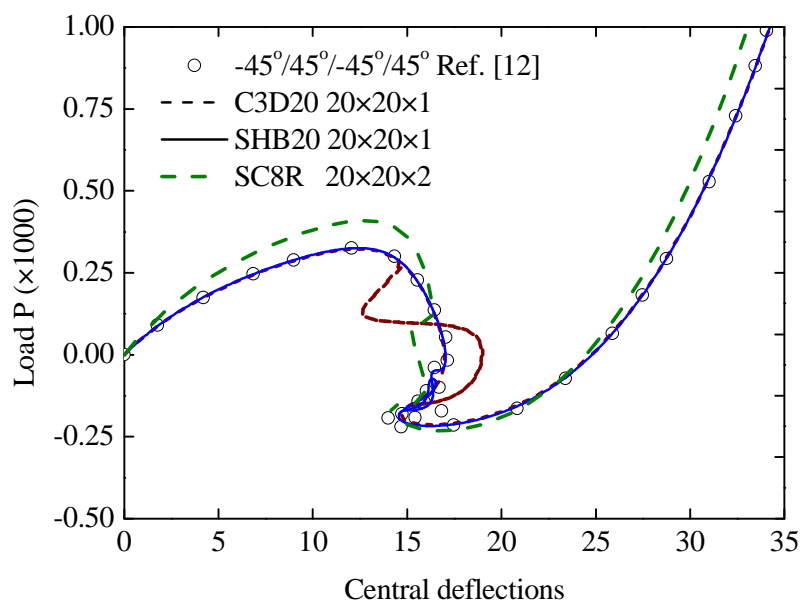

(d) hexahedral elements

Fig. 21. Load-vertical displacement curves at the central point A for the thin laminated cylindrical roof (i.e., $\left[30^{\circ} /-60^{\circ} /-60^{\circ} / 30^{\circ}\right]$ and $\left[-45^{\circ} / 45^{\circ} /-45^{\circ} / 45^{\circ}\right]$, with $\mathrm{t}=6.35$ ).

\subsection{Pull-out of an open-ended laminated cylindrical shell}

The last numerical example selected in this paper is a well-known benchmark problem consisting in a pull-out of an open-ended laminated cylindrical shell. As illustrated in Fig. 22, the open-ended thin cylindrical shell is pulled by a pair of radial forces, which results in large rotations and displacements during the stretching process (see the final deformed shape of the cylindrical shell in Fig. 23). Two different stacking sequences with respect to the circumferential direction are considered for the composite laminate, i.e., $\left[0^{\circ} / 90^{\circ}\right]$ and $\left[90^{\circ} / 0^{\circ}\right]$. The engineering constants of the orthotropic material are $E_{11}=3.05 \times 10^{7}, E_{22}=E_{33}=1.05 \times 10^{7}$, 
$v_{12}=v_{13}=v_{23}=0.3125, G_{12}=G_{13}=G_{23}=4 \times 10^{6}$. Owing to the symmetry of the problem, only one eighth of the cylinder is modeled.

Note that, due to the strong geometric nonlinearities involved in this benchmark problem, a fixed incrementation scheme is adopted instead of the default automatic scheme in order to obtain accurate results. Consequently, the NINC and NITER required by all finite elements are not shown for this test. The simulation results in terms of load-radial displacement curves at points A, B and C (as depicted in Fig. 22), which are obtained with the SHB and ABAQUS elements, are reported in Figs. 24 and 25 along with the reference solutions taken from [44, 45]. Once again, the proposed SHB elements successfully pass this benchmark test as compared to the reference solutions, which is also the case for the prismatic C3D15 ABAQUS element, although the latter requires two element layers in the thickness direction. For the hexahedral C3D20 ABAQUS element, however, some differences with respect to the reference solutions may be observed, especially in the transition zone marked by the snap-through point. As to the ABAQUS solidshell elements (i.e., SC6R and SC8R), the latter provide the farthest results as compared to the reference solutions (see Figs. 24 and 25).

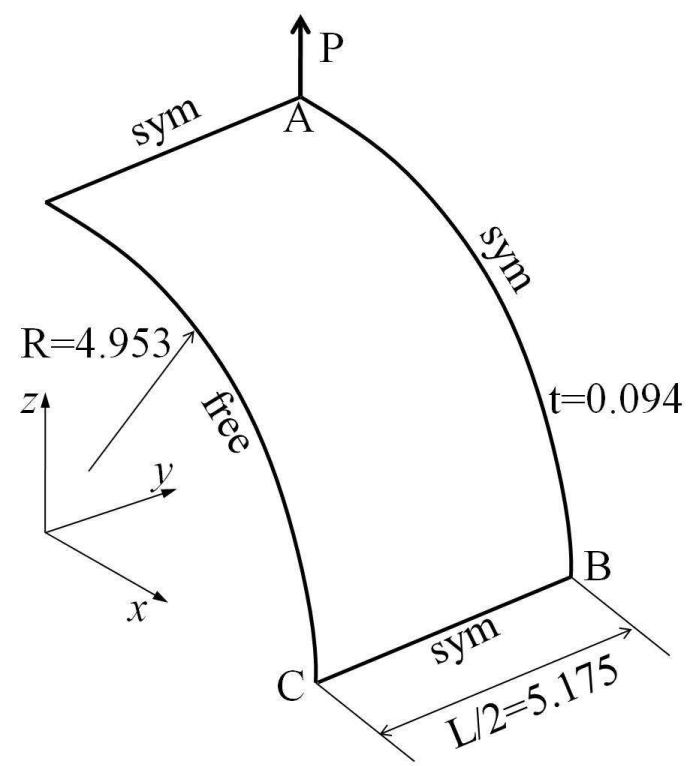

Fig. 22. Geometry and boundary conditions for the open-ended laminated cylindrical shell. 

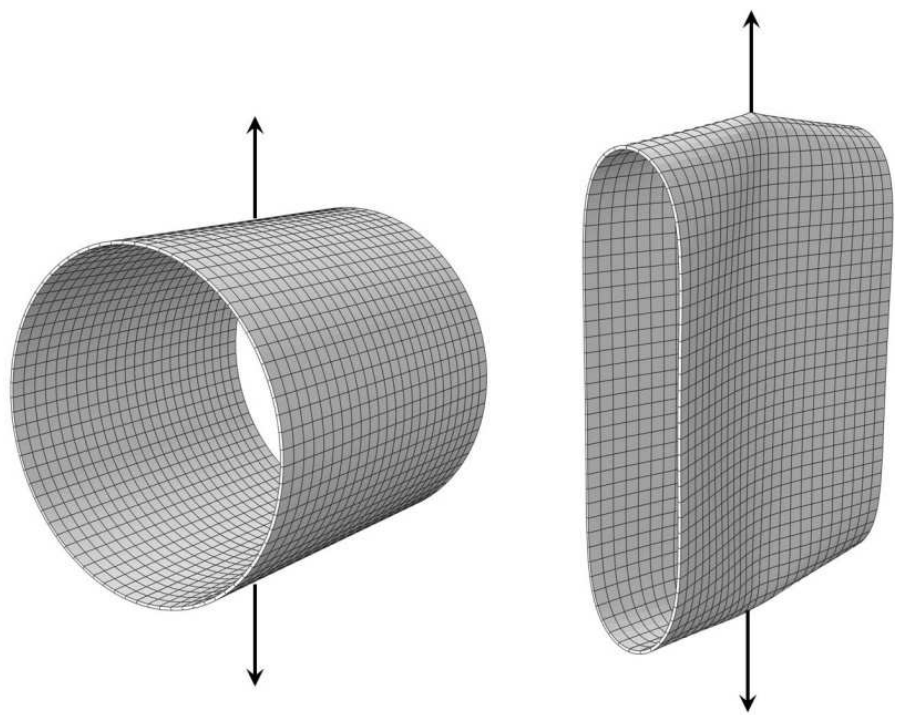

Fig. 23. Undeformed and final deformed shapes of the open-ended laminated cylindrical shell.

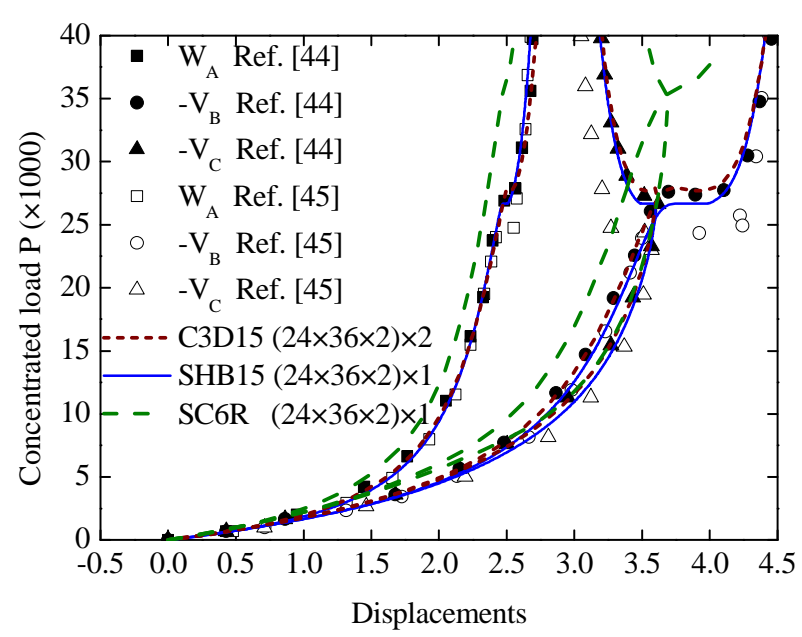

(a) prismatic elements

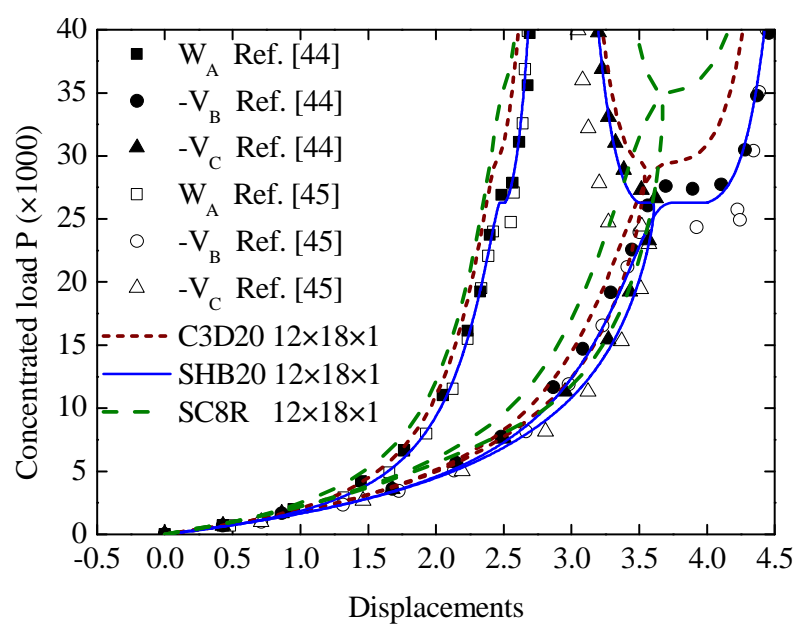

(b) hexahedral elements

Fig. 24. Load-radial displacement curves at points A, B and C for the open-ended laminated cylindrical shell with the stacking sequence $\left[0^{\circ} / 90^{\circ}\right]$. 


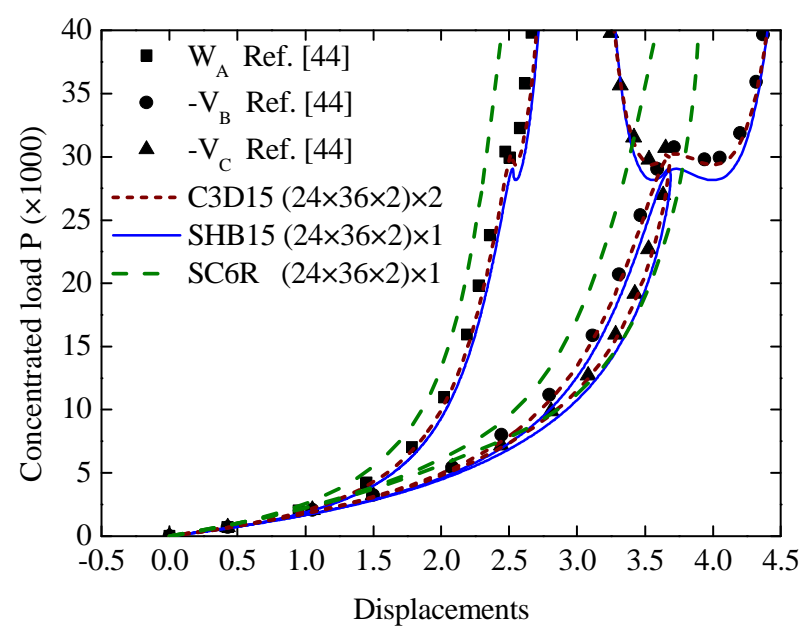

(a) prismatic elements

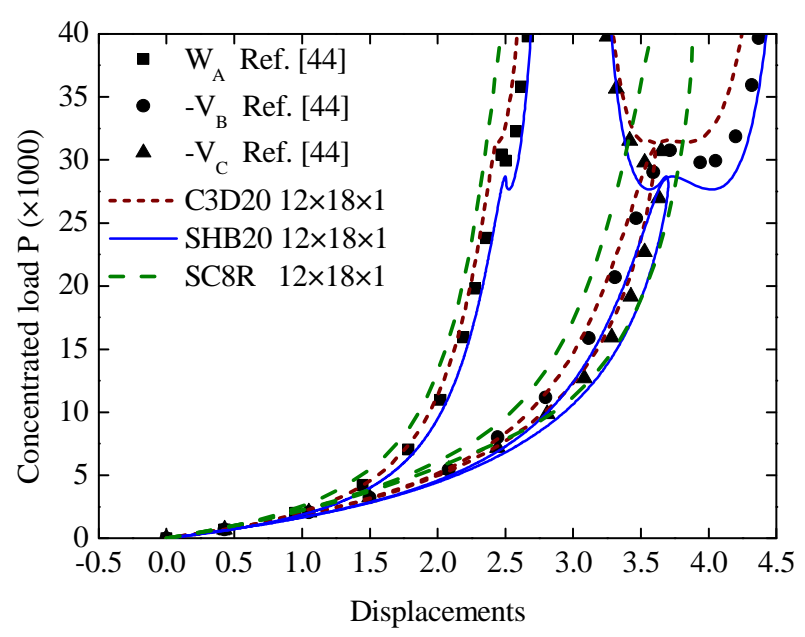

(b) hexahedral elements

Fig. 25. Load-radial displacement curves at points A, B and C for the open-ended laminated cylindrical shell with the stacking sequence $\left[90^{\circ} / 0^{\circ}\right]$.

\section{Conclusions}

In this paper, two quadratic (prismatic and hexahedral) assumed-strain based solid-shell elements, denoted as SHB15 and SHB20, respectively, have been proposed for the threedimensional modeling of multilayered composite structures. Following the earlier works on the family of SHB elements, the formulation of these solid-shell elements is extended in this work to the geometric nonlinear analysis of laminated composite materials. These elements are based on a fully three-dimensional framework, with only translational degrees of freedom, which allows accounting for the various through-thickness phenomena without the well-known restrictions associated with plane-stress assumptions. The resulting SHB elements, which have been implemented into the finite element code ABAQUS/Standard, are able to model multilayered composite structures using only a single element layer through the thickness. The performance of the proposed quadratic solid-shell elements has been assessed through several popular benchmark problems for geometric nonlinear analysis of laminated composite structures. The simulation results reveal that the proposed solid-shell elements have very good accuracy with respect to the reference solutions, while the ABAQUS linear solid-shell elements as well as the ABAQUS quadratic solid elements show several limitations and inaccuracies in modeling severe nonlinear problems for composite structures. On the whole, the variety of numerical results 
presented in this work demonstrate the good capabilities of the SHB elements and their wide prospective applications in the simulation of 3D laminated composite structures with only a single element layer through the thickness.

\section{References}

[1] Srinivas S, Rao AK. Bending, vibration and buckling of simply supported thick orthotropic rectangular plates and laminates. Int J Solids Struct 1970;6:1463-1481.

[2] Pagano NJ. Exact solutions for composite laminates in cylindrical bending. J Compos Mater 1969;3:398-411.

[3] Sciuva MD. Bending, vibration and buckling of simply supported thick multilayered orthotropic plates: an evaluation of a new displacement model. J Sound Vib $1986 ; 105(3): 425-442$.

[4] Khdeir AA. Comparison between shear deformable and Kirchhoff theories for bending, buckling and vibration of antisymmetric angle-ply laminated plates. Compos Struct 1989;13:159-172.

[5] Chueng YK, Kong J. Approximate three-dimensional analysis of rectangular thick laminated plates: bending, vibration and buckling. Comput Struct 1993;47(2):193-199.

[6] Wang J, Liew KM, Tan MJ, Rajendran S. Analysis of rectangular laminated composite plates via FSDT meshless method. Int J Mech Sci 2002;44:1275-1293.

[7] $\mathrm{Wu} \mathrm{Z}$, Chen WJ. Free vibration of laminated composite and sandwich plates using global-local higher-order theory. J Sound Vib 2006;298:333-349.

[8] Wagner W, Gruttmann F. A simple finite rotation formulation for composite shell elements. Eng Computations 1994;11:145-176.

[9] Polit O, Touratier M. A multilayered/sandwich triangular finite element applied to linear and non-linear analysis. Compos Struct 2002;58:121-128.

[10] Khare RK, Kant T, Garg AK. Free vibration of composite and sandwich laminates with a higher-order facet shell element. Compos Struct 2004;65:405-418.

[11] Lee WH, Han SC. Free and forced vibration analysis of laminated composite plates and shells using a 9-node assumed strain shell element. Comput Mech 2006;39:41-58. 
[12] Arciniega RA, Reddy JN. Tensor-based finite element formulation for geometrically nonlinear analysis of shell structures. Comput Methods Appl Mech Engng 2007;196:1048-1073.

[13] Damil N, Potier-Ferry M. A new method to compute perturbed bifurcations: application to the buckling of imperfect elastic structures. Int J Engng Sci 1990;28:943-957.

[14] $\mathrm{Hu} \mathrm{H}$, Belouettar S, Potier-Ferry M, Makradi A. A novel finite element for global and local buckling analysis of sandwich beams. Compos Struct 2009;90:270-278.

[15] $\mathrm{Hu} \mathrm{H}$, Belouettar S, Potier-Ferry M, Makradi A, Koutsawa Y. Assessment of various kinematic models for instability analysis of sandwich beams. Engng Struct 2011;33:572-579.

[16] Nguyen-Van H, Mai-Duy N, Karunasena W, Tran-Cong T. Buckling and vibration analysis of laminated composite plate/shell structures via a smoothed quadrilateral flat shell element with in-plane rotations. Comput Struct 2011;89:612-625.

[17] Payette GS, Reddy JN. A seven-parameter spectral/hp finite element formulation for isotropic, laminated composite and functionally graded shell structures. Comput Methods Appl Mech Engng 2014;278:664-704.

[18] Choi IH. Geometrically nonlinear transient analysis of composite laminated plate and shells subjected to low-velocity impact. Compos Struct 2016;142:7-14.

[19] Yu K, Hu H, Tang HY, Giunta G, Poiter-Ferry M, Belouettar S. A novel two-dimensional finite element to study the instability phenomena of sandwich plates. Comput Methods Appl Mech Engng 2015;283:1117-1137.

[20] Yang J, Huang Q, Hu H, Giunta G, Belouettar S, Potier-Ferry M. A new family of finite elements for wrinkling analysis of thin films on compliant substrates. Compos Struct 2015;119:586-577.

[21] Carrera E. Theories and finite elements for multilayered plates and shells: a unified compact formulation with numerical assessment and benchmarking. Arch Comput Methods Engng 2003;10(3):215-296.

[22] Huang Q, Liu Y, Hu H, Shao Q, Yu K, Giunta G, Belouettar S, Potier-Ferry M. A Fourier-related double scale analysis on the instability phenomena of sandwich plates. Comput Methods Appl Mech Engng 2017;318:270-295. 
[23] Liao CL, Reddy JN, Engelstad SP. A solid-shell transition element for geometrically nonlinear analysis of laminated composite structures. Int $\mathbf{J}$ Numer Meth Engng 1988;26:1843-1854.

[24] Klinkel S, Gruttmann F, Wagner W. A continuum based three-dimensional shell element for laminated structures. Comput Struct 1999;71:43-62.

[25] Vu-Quoc L, Tan XG. Optimal solid shells for non-linear analysis of multilayer composites. I. Statics. Comput Methods Appl Mech Engng 2003;192:975-1016.

[26] Quy ND, Matzenmiller A. A solid-shell element with enhanced assumed strains for higher order shear deformations in laminates. Tech Mech 2008;28(3-4):334-355.

[27] Naceur H, Shiri S, Coutellier D, Batoz JL. On the modeling and design of composite multilayered structures using solid-shell finite element model. Finite Elem Anal Des 2013;70-71:1-14.

[28] Hajlaoui A, Jarraya A, El Bikri K, Dammak F. Buckling analysis of functionally graded materials structures with enhanced solid-shell elements and transverse shear correction. Compos Struct 2015;132:87-97.

[29] Kpeky F, Boudaoud H, Abed-Meraim F, Daya EM. Modeling of viscoelastic sandwich beams using solid-shell finite elements. Compos Struct 2015;133:105-116.

[30] Abed-Meraim F, Combescure A. SHB8PS-a new adaptive, assumed-strain continuum mechanics shell element for impact analysis. Comput Struct 2002;80:791-803.

[31] Abed-Meraim F, Combescure A. An improved assumed strain solid-shell element formulation with physical stabilization for geometric non-linear applications and elasticplastic stability analysis. Int J Numer Meth Engng 2009;80:1640-1686.

[32] Trinh VD, Abed-Meraim F, Combescure A. A new assumed strain solid-shell formulation "SHB6" for the six-node prismatic finite element. J Mech Sci Tech 2011;25:2345-2364.

[33] Abed-Meraim F, Trinh VD, Combescure A. New quadratic solid-shell elements and their evaluation on linear benchmark problems. Computing 2013;95:373-394.

[34] Wang P, Chalal H, Abed-Meraim F. Efficient solid-shell finite elements for quasi-static and dynamic analyses and their application to sheet metal forming simulation. Key Engng Mat 2015;651-653:344-349.

[35] Hallquist JO. Theoretical manual for DYNA3D. Report UC1D-19041, Lawrence Livermore National Laboratory, Livermore, CA, 1983. 
[36] Simo JC, Hughes TJR. On the variational foundations of assumed strain methods. J Appl Mech 1986;53:51-54.

[37] Putcha NS, Reddy JN. A refined mixed shear flexible finite element for the nonlinear analysis of laminated plates. Comput Struct 1986;22(4):529-538.

[38] Kant T, Kommineni JR. C0 finite element geometrically non-linear analysis of fiber reinforced composite and sandwich laminates based on a higher-order theory. Comput Struct 1992;45(3):511-520.

[39] Buechter N, Ramm E. Shell theory versus degeneration-a comparison in large rotation finite element analysis. Int J Numer Meth Engng 1992;34:39-59.

[40] Brank B, Peric D, Damjanic FB. On implementation of a nonlinear four node shell finite element for thin multilayered elastic shells. Comput Mech 1995;16:341-359.

[41] Mohan P, Kapania RK. Updated Lagrangian formulation of a flat triangular element for thin laminated shells. AIAA J 1998;36:273-281.

[42] Sze KY, Liu XH, Lo SH. Popular benchmark problems for geometric nonlinear analysis of shells. Finite Elem Anal Des 2004;40:1551-1569.

[43] Andrade LG, Awruch AM, Morsch IB. Geometrically nonlinear analysis of laminate composite plates and shells using the eight-node hexahedral element with one-point integration. Compos Struct 2007;79:571-580.

[44] Kreja I, Schmidt R. Large rotations in first-order shear deformation FE analysis of laminated shells. Int J Non-Linear Mech 2006;41:101-123.

[45] Masud A, Tham CL, Liu WK. A stabilized 3-D co-rotational formulation for geometrically nonlinear analysis of multi-layered composite shells. Comput Mech 2000;26:1-12. 